\title{
Effects of Lexical Cues on Phrase Structure Encoding: Evidence from the Production of Genitives in Dutch
}

Chi Zhang ${ }^{\text {a,b }}$ iD https://orcid.org/0000-0003-4310-7417

Sarah Bernolet ${ }^{\mathrm{c}}$ iD https://orcid.org/0000-0002-3665-9750

Robert J. Hartsuiker ${ }^{a}$ (D https://orcid.org/0000-0002-3680-6765

${ }^{a}$ Department of Experimental Psychology, Ghent University, Ghent, Belgium;

${ }^{b}$ School of Foreign Languages and Cultures, Nanjing Normal University, Nanjing,

China;

${ }^{c}$ Department of Linguistics, University of Antwerp

Address for correspondence: Chi Zhang

Department of Experimental Psychology

Ghent University

Henri Dunantlaan 2

B-9000 Gent

Belgium

Tel. +32 (0)465 386530

chi.zhang@ugent.be 


\section{Abstract}

This paper examined the role of lexical processing in phrase structure building in sentence production. We asked whether speakers exploit a lexical cue as a lexical guide (i.e., the cued word occurs earlier in the sentence) and as a retrieval cue (i.e., a cue facilitates the retrieval of a memorized structure). In two experiments, participants recalled Dutch genitive sentences. In some recall trials, they received a lexical cue that repeated one argument of the to-be-recalled sentence. In two further experiments, participants read a genitive sentence and then generated a new one from a visually-presented triplet of arguments. The visual salience of the arguments and lexical overlap were manipulated. In all four experiments, speakers consistently started the phrase with the cued word. There was no evidence of a lexical cueing effect on structure retrieval. The findings suggest that speakers mainly exploit lexical information as a lexical guide when formulating phrase structures.

Keywords: sentence recall; structural priming; sentence structure memory; lexical cueing 


\section{Introduction}

In our daily conversations, speakers often vary in their choices of sentence structures. For example, when asked, between Albert Einstein and Forrest Gump, whose IQ is higher, the speaker might say Albert Einstein's $I Q$ is higher, or they might alternatively say the IQ of Albert Einstein is higher. Such syntactic flexibility in sentence production can be influenced by multiple factors. This paper examines how lexical processing modulates syntactic choices in sentence production.

When we produce a sentence, we translate thoughts into meaningful strings of words. This process requires us to retrieve lexical items and formulate syntactic structures that are appropriate to express the intended concepts and that are comprehensible to listeners. Most models of language production assume that lexical representations and syntactic structures are computed and represented at the same stage of the language production system (Bock \& Levelt, 1994). Although syntactic processing can be independent of lexical retrieval (Bock \& Loebell, 1990; Ivanova, Pickering, Branigan, McLean, \& Costa, 2012), many linguistic theories and psycholinguistic models argue that lexical information is linked to syntactic structure (Bock, 1982; Bock \& Levelt, 1994; Ferreira \& Dell, 2000; Kaplan \& Bresnan, 1995; Levelt, 1989; Levelt, Roelofs, \& Meyer, 1999; Levin, 1993). Understanding grammatical encoding processes in sentence production thus requires an investigation of how speakers engage lexical information when formulating sentence structures. When a word is retrieved during sentence planning, multiple types of lexical information are available to speakers, which differentially determine the outcome of sentence structure formulation. For example, if a word is more identifiable in lexical retrieval, speakers tend to place that word earlier in the sentence. 
Meanwhile, the lexical item links to the syntactic structures it licenses. The accessing of the lexical item leads to the activation of these structures, which might also modulate the syntactic choice. How do speakers coordinate these different lexical effects in sentence production? Here we address this question by examining how speakers exploit a lexical cue when making syntactic choices in sentence production.

\section{Lexical Information Integration in Grammatical Encoding}

Lexically-specific information is stored in long-term memory and can be retrieved upon activation (Levelt et al., 1999). A lexical item can represent both non-combinatorial and combinatorial information (Boland \& Tanenhaus, 1991). For example, when accessing the verb chases, some types of non-combinatorial information such as the phonological description ([t $\mathrm{fe}$ eIsis $])$ and the orthographic form of the verb (chases) as well as some types of combinatorial information such as the syntactic category (verb), tense (present), number (singular), and subcategorization frames (e.g. [NP_NP], meaning chases can be linked to a frame containing two noun phrase arguments) are activated. In line with a lexicalist model of production (Levelt et al., 1999), the non-combinatorial information is indicative of the non-relational identification of the word, while the combinatorial information is indicative of the semantic or syntactic relation of the word to the rest of the components in the sentence. The combinatorial properties of a word are represented as nodes that link to the syntactic form of the word (i.e., a lemma).

What role does lexical information play in grammatical encoding? The construction of the syntactically related properties of the sentence is often conceptualized as a two-stage model (Bock \& Levelt, 1994; Bock, Loebell, \& Morey, 1992; Garrett, 1989; but see 
Pickering, Branigan, \& McLean, 2002 for a view of a one-stage model) in which concepts primarily map onto a hierarchical structure that encompasses their grammatical function (e.g., subject, object). This model assumes separate processing stages of function assignment and position assignment. Specifically, speakers first compute the gist of the preverbal message. This is then combined with the grammatical functions of the sentence constituents. In this stage, the lexical concepts and lemmas are also identified and retrieved. The outcomes of the binding (i.e., functionally "tagged" words) then enter the positional stage in which words are assembled into phrases and linear order is determined. This model assumes that lexical selection takes place in parallel with function assignment. The role of lexical selection in grammatical encoding would be to control the construction of the syntactic structure on the basis of the subcategorization frame that is linked to the lemma.

This view was further developed by Pickering and Branigan (1998). They proposed that the lexical-specific subcategorization frames are represented by 'combinatorial nodes' that link to the lemma node. Once a lexical item is activated, the activation from the lemma node spreads to the combinatorial node. For some verbs (e.g., give) more than one combinatorial node can be activated, but the levels of activation differ because of the lexical-specific preference (Ferreira, 1994; Lombardi \& Potter, 1992; Melinger \& Dobel, 2005) or short-term activation by recent linguistic experience (Cleland \& Pickering, 2003; Pickering \& Branigan, 1998). The more activated combinatorial node is then selected and determines the outcome of syntactic planning (i.e., syntactic choice).

Previous studies have shown that speakers' syntactic choice is influenced by a subcategorization preference that is inherent to the verb (Ferreira, 1994; Lombardi \& 
Potter, 1992; Stallings, MacDonald, \& O'Seaghdha, 1998). For example, Lombardi and Potter (1992) found that the speakers' wordings in sentence recall were susceptible to the intrusion of a lure word. For example, they were likely to replace the verb give from the to-be-recalled sentence with the lure word donate. Crucially, when the speakers did so, they also restored the sentence in such a way that the structure was compatible with the lure word. Suppose the to-be-recalled sentence was a double object sentence with the dative verb give (e.g., The rich widow is going to give the university a million dollars). When the speakers replaced give with the lure word donate, they were more likely to reconstruct a prepositional object sentence (The rich widow is going to donate a million dollars to the university), because in English, the prepositional object is the only dative structure that is licensed by donate. This leads to the argument that the production process in sentence recall is susceptible to the syntactic privileges posited by a recently activated lexical item.

Some of the strongest evidence for the modulation of lexical-specific syntactic preference on syntactic decision comes from studies on the lexical boost effect of structural priming (Branigan, Pickering, \& Cleland, 2000; Cleland \& Pickering, 2003; Hartsuiker, Bernolet, Schoonbaert, Speybroeck, \& Vanderelst, 2008; Pickering \& Branigan, 1998). Studies on structural priming showed that speakers have the tendency to spontaneously reuse the syntactic choice of a previously experienced sentence (see Mahowald, James, Futrell, \& Gibson, 2016 for a meta-analysis of structural priming in production). Many of these studies have shown that syntactic persistence is independent of the overlap in semantic or prosodic representations (Bock \& Loebell, 1990; Bock et al., 1992; Scheepers, 2003; but see Ziegler, Bencini, Goldberg, \& Snedeker, 2019 for a non-abstract view of structural 
priming). Nevertheless, it was found that lexical overlap considerably enhances the magnitude of structural priming (i.e., lexical boost). Pickering and Branigan (1998) demonstrated that the priming effect was much larger when the verb of a target sentence completion task (e.g., show in The patient showed...) matched with the verb in a prime sentence completion task (e.g., show in The racing driver showed the helpful mechanic...) relative to when the verbs were unmatched (e.g., give-show). The lexical boost effect can be explained by the lexicalist model of production (Pickering \& Branigan, 1998) in that the lexical-specific subcategorization frame activated in the prime task persists to the target task and biases speakers to use the primed structure in the ensuing production. Note that apart from the studies that showed a lexical boost effect on syntactic choice between clausal structures, the lexical overlap was also found to promote the choices of phrasal structures (Bernolet, Hartsuiker, \& Pickering, 2013; Cleland \& Pickering, 2003; Konopka \& Bock, 2009). This indicates that the lexical-specific combinatorial representation would also influence the constituent encoding at the phrasal level.

Apart from the two-stage architecture, another fundamental feature of the grammatical encoding process is that it is incremental: speakers often initiate the utterances without a fleshed-out plan for every aspect of the sentence. This requires them to optimize resources during sentence planning. A good way to facilitate efficient speech production is to arrange the timing of information processing on the basis of information accessibility. If a lexical item is more identifiable at the outset of sentence production, the lemma node of the lexical item becomes more accessible to the speakers. In order not to add more to the computational load of sentence planning by holding the accessed word in 
memory for too long, speakers tend to retrieve the more accessible lemma as soon as possible (Ferreira \& Dell, 2000). The early selected lemma then guides the construction of the relational structure for the rest of the sentence. This lexical-specific guidance on syntactic choice is often termed the guidance effect (Vigliocco \& Hartsuiker, 2002).

The notion of the guidance effect we discussed here should be distinguished from the notion of conceptual accessibility effect (see Bock, Irwin, \& Davidson, 2004 for a discussion of the distinction between the two). Studies on conceptual accessibility effects stressed that lexical concepts with higher prominence (e.g., more animate, imaginable, or familiar) are easier to be mapped onto the hierarchically highest argument (e.g., subject in English; the hierarchy of functions is based on linguistic analysis, Kaplan \& Bresnan, 1995). The conceptual accessibility only plays a role when formulating the conceptual relation between the arguments, so it should only modulate the subsequent function assignment process (Bock \& Irwin, 1980; Bock \& Warren, 1985; McDonald, Bock, \& Kelly, 1993; but see Prat-Sala \& Branigan, 2000). In contrast, the lexical guidance effect often refers to the effect that the perceptually more prominent items are easier to be identified and subsequently lead to an early retrieval and placement in sentence formulation. This guidance effect mainly modulates phrase structure order, but it can also influence the speakers' choice of clause structures (Gleitman, January, Nappa, \& Trueswell, 2007; Myachykov, Garrod, \& Scheepers, 2009)

Studies on the guidance effect, especially those that investigated production in a nonGermanic language, showed that the more accessible item directly influences word order (Christianson \& Ferreira, 2005; Ferreira \& Yoshita, 2003; Myachykov, Garrod, \& Scheepers, 2010; Myachykov \& Tomlin, 2008; Prat-Sala \& Branigan, 2000; Tanaka, 
Branigan, McLean, \& Pickering, 2011). For example, Myachykov and Tomlin (2008)

found that when native Russian speakers described a transitive event, an attentioncapturing cue that highlighted one referent of the event led to an early placement of the cued referent in sentence production. Similarly, Prat-Sala and Branigan (2000) found that English and Spanish speakers were more likely to start the sentence with a referent that was more salient in the preceding discourse. More importantly, they found that when the object was made salient, Spanish speakers also showed a greater tendency to front the object in the sentence, rendering a dislocated active (e.g., A la mujer la atropelló el tren. [Literally: To the woman she ran over the train], meaning the train ran over the woman). Taken together, these findings indicate that perceptually salient items tend to appear at the linearly first position.

Further evidence for a guidance effect comes from studies on phrase structure choices (Gleitman et al., 2007; Kelly, Bock, \& Keil, 1986). Constituents such as conjoined noun phrases (conjoined NPs, e.g., A dog and a cat/ a cat and a dog) usually take a fixed grammatical role (subject or object) applying to both constituent nouns, but the word order or the thematic order within the constituent is flexible. Gleitman and colleagues (2007) asked native English speakers to describe, among others, an event that involves conjoined NPs while manipulating a subtle visual cue that directed speakers' gaze to certain referents on the picture. Speakers' choice of the initial NP was modulated by the location of visual cues: They were more likely to begin their sentences with the character to which their eye had been guided. This further suggests that speakers' choices of phrase structure can be guided by the perceptually more salient words at the outset of speech.

So far, we have reviewed two essential roles of lexical information in grammatical 
encoding. Speakers' syntactic choice is modulated by the activation of the subcategorization structure linked to a lemma. This is the case for instance in the lexical boost of structural priming. Additionally, the construction of syntactic structure can be guided by the more accessible lexical item, resulting in a guidance effect. When a lemma is accessed, both information about accessibility and the lexical-specific subcategorization is available to the speakers. These two types of information might point the speaker toward the same syntactic choice, but on other occasions, they may promote different options in syntactic selection. Consider the example of genitive phrase structures again. Speakers can choose between the phrase structure of Albert Einstein's IQ is higher (s-genitive) or the IQ of Albert Einstein is higher (of-genitive). But if the referent $I Q$ is made more salient during lexical access (e.g., by discussing IQ in the previous context), the speakers would be biased to put $I Q$ at the beginning of the sentence, which renders an of-genitive sentence. However, if the s-genitive structure that links to the lemma $I Q$ happens to be activated by a prime sentence, the lexical boost effect would bias the speakers to the s-genitive structure. How do speakers adapt their syntactic choice to the lexical specific information when this information activates competing phrase structures? The goal of the current study is to examine how speakers resolve the conflict between these lexical effects when making syntactic choices between alternative genitive phrase structures.

\section{Rational Coordination of Lexical Information in Grammatical Encoding}

Language production is often argued to be a system in which speakers have to choose between alternatives at multiple linguistic levels that are equally felicitous (Dell \& O'Seaghdha, 1994; Myachykov, Scheepers, Garrod, Thompson, \& Fedorova, 2013; see 
Melinger, Branigan, \& Pickering, 2014 for a discussion). Specific to the syntactic flexibility in sentence production, speakers prepare several syntactic alternatives in parallel. The final selection among those options relies upon multiple constraints (e.g., Ferreira, 1994; Myachykov, Garrod, \& Scheepers, 2012; Prat-Sala \& Branigan, 2000; Stallings et al., 1998). In many cases, these constraints might not work in concert. For instance, Ferreira (1994) found in a series of sentence generation tasks that the mandatory use of a theme-experiencer verb (e.g. challenge) predisposed speakers to produce a passive sentence (e.g., The cowboy was challenged by the frontier). A homogeneous distribution of animacy between arguments (e.g., the arguments are animate nouns cowboy and sheriff), however, led to more active sentences (e.g., The cowboy avoided the sheriff) in such cases, relative to a heterogeneous distribution of animacy (e.g., one argument is an animate noun cowboy and the other is an inanimate noun frontier). Given that speakers can only select one structure in the end and that the blending of two syntactic options leads to a speech error, syntactic encoding in sentence production sometimes entails a trade-off between different constraints (see MacDonald, 2015 for a related discussion). One intriguing question is how speakers coordinate these constraints. More specifically, in what way do speakers assess and consider different types of information when they make syntactic choices? A recent view of sentence production, adapted from the competition model of language processing (Bates \& MacWhinney, 1982, 1989), posited that speakers rationally adapt the formulation of sentence structure to the information they experienced (e.g., Deutsch \& Dank, 2009; Perek \& Goldberg, 2017; Thothathiri, 2021; Thothathiri \& Braiuca, 2021; Thothathiri \& Rattinger, 2016; also see Haskell \& MacDonald, 2003). Two of the important tenets of such a view 
(hereafter referred to as "the rational view") are that sentence production is competitive and rational. Essentially, the encoding of a sentence entails the competition between multiple constraints. The constraints that are weighted more tend to block the effectiveness of the constraints that are weighted less. The relative weights of these constraints are rationally assigned based on the predictive reliability, which corresponds to the statistical contingencies in which a constraint can be mapped onto a specific outcome.

Previous studies showed that speakers indeed tune their syntactic choice to the reliability of the constraints (Perek \& Goldberg, 2017; Thothathiri, 2021; Thothathiri \& Braiuca, 2021; Thothathiri \& Rattinger, 2016). Many of these studies investigated how speakers coordinate lexical-specific biases (i.e., the mapping between a verb and a syntactic structure) and lexical-general semantic constraints (i.e., the mapping between an event and a syntactic structure) when formulating syntactic structures in their native language or an artificial language. For example, Thothathiri and Rattinger (2016) trained their participants in an artificial language with two syntactic structures: Verb-Agent-Patient transitive (e.g., pelk zebra giraffe, meaning a zebra jumps on a giraffe) and Verb-PatientAgent transitive (e.g., fenk giraffe lion ka, meaning a lion kisses a giraffe). They varied the verb bias of the artificial language such that some of the verbs were exclusively predictive of one structure and some alternated between the two structures. This made the lexical-specific biases a highly (but not $100 \%$ ) reliable constraint. The authors found that speakers' syntactic choices in a post-learning production task were sensitive to the variation of verb bias. Importantly, in the subsequent experiments that trained the participants with a similar syntactic alternation, the authors increased the reliability of the 
lexical-general semantic constraint: Instead of fully crossing the event semantics with the structures as in the first experiment, the instrument events (e.g., a monkey pinches a giraffe using a clip) were only mapped onto the Verb-Agent-Patient-Object structure whereas the modifier events (e.g., a monkey scratches a donkey that holds the paper on the back) were only mapped onto the Verb-Patient-Agent-Object structure. This way, the event semantics was $100 \%$ predictive of the structure and thus had a higher reliability than the lexical-specific constraint. Thothathiri and Rattinger found that in these experiments, the lexical-specific bias no longer affected speakers' syntactic choice. Speakers exclusively utilized event semantics as a constraint to plan the upcoming syntactic structure. Taken together, the findings suggest that speakers flexibly adapt the structural formulation to the information they experienced based on how predictive this information is of a specific structure. Additionally, the findings in Thothathiri and Rattinger, among other studies, suggest that the competition between constraints is not only limited to the condition where the constraints elect different syntactic alternatives. The competition is rather across-the-board in that the blocking effect from the more reliable constraint can permeate the conditions where the constraints show no conflict.

Following the rational view of sentence production, we assume that speakers can rationally select syntactic structures based on the predictive strength of the constraints. If this is the case, the lexical overlap on the one hand and the thematic-role-specific accessibility on the other hand can be taken as two constraints that competitively determine the syntactic choice. Which constraint wins this competition hinges on how reliably each constraint predicts a specific structure. If the thematic-role-specific distribution of accessibility unfailingly leads to one syntactic outcome (e.g., the more 
accessible word $I Q$ always results in the $I Q$ of Einstein) while the lexical overlap associates with multiple syntactic options in a more balanced way (e.g., when the word $I Q$ is repeated across utterances, it either signals sentences like the IQ of Einstein or Einstein's $I Q$ ), we can assume the lexical guidance has a higher reliability. This way, the guidance effect might block the effect of the lexical boost. But the lexical boost will win this competition if the opposite is true.

We further asked whether the coordination of lexical information processing can be modulated by other cognitive-general factors such as attentional status (i.e., to what extent speakers shift their focus to certain aspects of the linguistic experience). Studies in structural priming (e.g., Bock et al., 1992) showed that speakers' sentence encoding is modulated by the attentional resources that speakers assign to processing certain levels of information: When the participants' attention was directed to the semantic aspects of the prime sentence, they showed a stronger tendency to mimic the assignment of animate vs. inanimate concepts to the grammatical functions of subject and object, while there was a stronger structural priming effect when their attention was directed to the syntactic form of the prime. Similarly, we hypothesized that if speakers attended more to the sentence structure throughout the process of sentence encoding and production, they would prioritize the structure retrieval in grammatical encoding and rely more on a constraint related to a lexical-specific subcategorization frame. It is not clear how attentional status interplays with the rational adaptation in sentence production. It is possible that the attentional shift could override the competition between constraints whereby it elects the less reliable but more attended constraint as an influential factor that determines syntactic encoding. 
We conducted four experiments to test the above predictions. In all experiments, we manipulated the accessibility of the referents in the production of the target, the prime structure, and lexical overlap of the syntactic head. We first employed two sentence recall tasks. In these tasks, speakers memorized and recalled Dutch genitive constructions (sgenitive: De heks haar eend is rood [Literally: The witch her duck is red] vs. of-genitive: De eend van de heks is rood [Literally: The duck of the witch is red]). In half of the recall tasks, participants were presented with a lexical cue that either repeated the possesum (e.g., eend) or the possessor (e.g., heks) of the to-be-recalled sentence. In Experiment 1, we only cued the possessums. In Experiment 2, either the possessums or the possessors were cued. In Experiment 1 and 2, we also examined whether the appearance of a lexical cue facilitated the recall of the message (i.e., the possessive relations). This served as a test of the effectiveness of a lexical cue in sentence recall. Furthermore, in a structural priming experiment (Experiment 3 ) and a sentence structure memory experiment (Experiment 4), we aimed to replicate the lexical effects of Experiment 1 and 2 in a paradigm where all target arguments can be accessed during production but where they differ in visual prominence. In Experiment 4, the participants were explicitly instructed to pay attention to the syntactic structure of the prime sentence they experienced. This allowed us to evaluate how attention to genitive structures in the prime sentences might modulate these lexical effects in comparison with the effects in Experiment 3.

The genitive constructions in Dutch consist of two arguments: the object (i.e., the possessum) and the owner of the object (i.e., the possessor). Both constructions express possessive relations between the possessor and the possessum. The Dutch of-genitive is very much comparable to the of-genitive in English (e.g., The duck of the witch) whereas 
the Dutch s-genitive largely overlaps with the s-genitive in English (e.g., The witch's $d u c k$ ) but with certain distinctions. Different from the English s-genitive, the object of the construction is not morphologically marked by a bound morpheme (e.g., 's in English) ${ }^{1}$. Instead, a possessive pronoun (zijn or haar) is placed between the possessor and the possessum. The number and the gender of the pronoun agree with the possessor. Arguably, the possessive pronoun in a Dutch s-genitive has syntactic and semantic functions very similar to the bound morpheme in English s-genitive (Weerman \& De Wit, 1999). Indeed, previous studies have found cross-linguistic structural priming between the English s-genitive and the Dutch s-genitive, suggesting the compatibility of the formmeaning mapping between the constructions (Bernolet, Hartsuiker, \& Pickering, 2012; Bernolet et al., 2013).

Throughout the experiments, speakers' syntactic choices of genitive structures were assessed. As the genitive phrase takes a fixed grammatical function (i.e., subject) in the sentence, the alternation between the two structures does not involve the assignment of grammatical functions. This way, any lexical effect on genitive alternation should occur at the positional level. This allows us to examine the lexical effects on phrase encoding that arguably occurs at the positional level of grammatical encoding (Bock \& Levelt, 1994).

Although we did not manipulate the reliability of the constraint ad hoc, the reliabilities of the lexical overlap and the accessibility of a lexical item can be estimated. As we mentioned earlier, the reliability of the constraints in the current study corresponds to the extent to which the lexical overlap and the accessibility of the possessor/possessum predict a specific syntactic choice between the genitive alternatives as the experiment 
progresses. In terms of the lexical boost effect, the participants associated the repeated items with the s-genitive prime/to-be-recalled sentence in half of the trials, and with the of-genitive in the other half. Thus, when the lexical overlap occurred, there was $50 \%$ chance it would predict an s-genitive, and 50\% chance it would predict an of-genitive. This made the lexical overlap 50\% predictive of the genitive structures. Meanwhile, the predictive strength of the lexical guidance is arguably highly reliable. The guidance effect allows the speakers to formulate an almost one-to-one mapping between the more accessible words and the syntactic options. The more accessible word is consistently associated with the structure in which such a word comes first. The predictive reliability of the lexical accessibility is surely above the chance level and thus higher than that of the lexical overlap. Following the rational view of sentence production, we predict that when the two constraints occur simultaneously, the participants would exploit the constraint of lexical guidance rather than the lexical overlap in syntactic encoding. Thus, there would be significant effects of the guidance throughout the experiments, while the lexical boost effects might be at least weaker than the guidance effects. Since in the current experiments, the condition that enabled a lexical boost effect always co-occurred with the condition that enabled a guidance effect, we predict the lexical boost effect would be consistently attenuated by the guidance effect in all conditions. Nevertheless, a stronger lexical boost effect might manifest itself when the speakers attend more to the encoding and retrieval of the syntactic structure (i.e., in Experiment 4).

\section{Experiment 1}

In Experiment 1, we employed a sentence recall paradigm to examine how speakers exploit lexical cues in grammatical encoding. There has been a long tradition for 
researchers to use sentence recall in studies on syntactic choices in sentence production (Bock \& Brewer, 1974; Bock \& Irwin, 1980; Bock \& Warren, 1985; McDonald et al., 1993; Tannenbaum \& Williams, 1968). Potter and Lombardi (1992; 1998) argued that speakers produce sentences in a recall task by reconstructing syntactic structures. In such a regeneration process, speakers build up the syntactic structure of the to-be-recalled sentence based on the conceptual information they retrieve from explicit memory (i.e., the memory system that one can consciously draw upon to retrieve or recall the information). The syntactic choice of the speakers is not arbitrary. It is essentially modulated by the structural priming effect induced by the to-be-recalled sentence. If so, the persistence of syntactic structure in sentence recall may be promoted by extrinsic lexical cues. A cue that repeats the lexical content of the to-be-recalled sentence would lead to a stronger activation of the to-be-recalled content words, which subsequently enhances the activation of the to-be-recalled syntactic structures that were associated with the words. This would predict a higher chance of correct recall in the sentence recall tasks when a lexical cue is shown to the participants. In other words, a lexical cue in sentence recall would result in a lexical boost, similar to that in the structural priming experiments. There is no direct evidence for the effect of lexical cues on sentence structure formulation in sentence recall. Nevertheless, several studies on structural priming demonstrated that some lexical-specific effects of structural priming have analogies with memory effects (Branigan \& McLean, 2016; Hartsuiker et al., 2008; Man, Meehan, Martin, Branigan, \& Lee, 2019; Rowland, Chang, Ambridge, Pine, \& Lieven, 2012; Zhang, Bernolet, \& Hartsuiker, 2020). For example, lexical boost effects appear to rapidly decay after two filler trials (Branigan \& McLean, 2016; Hartsuiker et al., 2008) and they are susceptible 
to memory impairment (Man et al., 2019; Zhang et al., 2020). This implies that there is an explicit memory-related component in the lexical boost effects. More specifically, speakers temporarily store the surface structure and the wording of prime sentences in explicit memory. The encoded prime sentence can be retrieved in the subsequent production tasks so as to facilitate syntactic processing, thus contributing to the general structural priming effect. Arguably, speakers employ the repeated lexical item as a lexical cue to trace and reinstate the sentence structure, resulting in a higher chance to repeat the prime structures when there is lexical overlap between the prime and target. This presupposes that a lexical cue should facilitate the retrieval of a memorized sentence structure. This leads to the prediction that a lexical cue should also facilitate structure retrieval in a task where speakers' memory of the sentence is being taxed (e.g., a sentence recall task). On the other hand, displaying a lexical cue would of course make the cued word more available to the speakers in the recall tasks. This increase of accessibility likely modulates the order of arguments in grammatical encoding, biasing the speakers to put the cued word earlier in the sentence (Bock, 1986, 1987; Bock \& Irwin, 1980; Tannenbaum \& Williams, 1968).

Note that in Experiment 1, we only cued the possesssum of the sentence. This is to follow the convention in the literature of the lexical boost effect on structural priming (e.g., Branigan, Pickering, Stewart, \& McLean, 2000; Hartsuiker et al., 2008; Pickering \& Branigan, 1998). In most previous studies, only the syntactic head of the phrase was manipulated (c.f., Carminati, van Gompel, \& Wakeford, 2019; Scheepers, Raffray, \& Myachykov, 2017). Similarly, we only presented possessum cues to the participants, which we expect to predispose the participants to the of-genitive structure. 
Taken together, we assume that both non-combinatorial and combinatorial information associated with the lexical cue play a role in grammatical encoding during sentence recall. More specifically, the appearance of a possessum cue could increase the accuracy of the sentence structure recall, such that the participants would be more likely to produce an s-genitive structure after an s-genitive to-be-recalled sentence (i.e., the lexical boost effect). On the other hand, that same possessum cue might induce an overall increase of of-genitive production and subsequently reduce the overall likelihood to produce sgenitive sentences in the recall tasks (i.e., the guidance effect).

We have discussed the predictive reliabilities of lexical overlap and lexical guidance in the Introduction. To summarize, we predict lexical accessibility to be a more reliable constraint than lexical overlap and thus might block the functionality of the lexical overlap. In addition, we believe that in the sentence recall paradigm, there is at least one extra factor that might further weaken the lexical boost effect: the interference between structure encoding and retrieval. Experiments in sentence recall usually place filler trials in between sentence encoding and retrieval in order to avoid a potential ceiling effect (Konopka \& Bock, 2009; Lombardi \& Potter, 1992; Potter \& Lombardi, 1998). The recall tasks used in the current study followed this tradition: We put two filler trials between sentence encoding and retrieval. This would exert an interference effect on the storage of memory traces including the memory of the sentence structure. Arguably, the weaker memory of sentence structure might subsequently lead to a reduced lexical boost effect (Branigan \& McLean, 2016; Hartsuiker et al., 2008; Man et al., 2019).

In addition to the lexical boost effect and the guidance effect, the access to the cue might facilitate the reinstatement of the to-be-recalled message. It has been suggested that the 
recall of messages can be facilitated by a lexical cue that partially reinstates the lexical or conceptual representation of the to-be-recalled sentence (Masson, 1979; Till, 1977). In our sentence recall experiments, we also examine the cueing effect on the recall of the possessive relations in order to examine whether the lexical cue can be taken as a retrieval cue at all in sentence recall.

\section{Method}

\section{Participants}

Forty-one Ghent University students, all native Dutch speakers, participated in exchange for course credit (29 females and 12 males, average age 19.15 years). All participants reported to be non-color-blind and had normal or corrected-to-normal vision.

\section{Materials and Design}

Figure 1 illustrates the composition of tasks in an experimental trial. Each trial started with a sentence memorization task, followed by a picture verification task, a picture description task, a sentence reading task, a picture verification task, and ended with a sentence recall task (in which the cue was or wasn't given). Among the trials in each experiment list, the trials that started with a genitive sentence memorization task were critical trials, and the rest were filler trials. For the sentence memorization task and the sentence reading task, we created 320 sentences (160 genitive sentences and 160 transitive sentences). The critical sentences in Experiment 1 and all subsequent experiments are available online (https://osf.io/yqwsj/). The genitive sentences comprised eighty pairs. The two sentences in each pair expressed the same meaning but differed in 
the constituent structure of the genitive noun phrases. One sentence was in the s-genitive structure (1a, the underlined word was used as a lexical cue in the Lexical cue condition) and the other one was in the of-genitive structure (1b). Half of these genitive pairs (40 sgenitive and 40 of-genitive) were employed as the sentences that the participants had to memorize (i.e., to-be-recalled sentences) in the sentence memorization task in the critical trials. The rest were employed as the sentences that the participants had to read in the sentence reading task in the filler trials. This yielded 40 genitive to-be-recalled sentences for each experiment list. Forty recall cues were selected for the genitive to-be-recalled sentences, namely the possessums of the sentences (e.g., eend [duck]).

1a) De heks haar eend is rood [Literally: The witch her duck is red]

1b) De eend van de heks is rood [Literally: The duck of the witch is red]

[Figure 1 near here]

Similarly, the transitive sentences, which were taken as filler stimuli, were 80 sentence pairs that were composed of an active sentence (e.g., De politieagent scheldt de ballerina uit [The policeman scolds the ballet dancer]) and a PP-final passive sentence (e.g., De ballerina wordt uitgescholden door de politieagent [The ballet dancer is scolded by the policeman]). Half of the pairs (40 active, 40 passive) were employed in the sentence recall tasks in the filler trials, and the rest were applied to the sentence reading tasks in the critical trials. Forty cues were created for the transitive to-be-recalled sentences that repeated the agent/patient of the sentences (e.g., politieagent [policeman]/ ballerina [ballet dancer]). 
We created a set of 160 pictures for the picture verification tasks. The events depicted in these pictures were derived from the sentences introduced above. Eighty of them were pictures that depicted genitive relations (e.g., a red duck that belongs to a witch, see the example of the first verification picture in Figure 1), and the rest were pictures that depicted transitive events (e.g., a train is running over a bus, see the example of the second verification picture in Figure 1). Half of the genitive pictures were employed in the verification task for the to-be-recalled sentences in the critical trials and the other were employed in the verification task for the reading stimuli in the filler trials. On each genitive picture, the figurines were holding an object, wearing an object, or standing next to an object, indicating the status of ownership. One object in the picture was colored. The rest of the picture was in black and white. All the figurines in the participant's verification set were chosen equally often from a boy, a girl, a nurse, a wizard, a pirate, a nun, a priest, and a witch. The colored object was filled by one of the four colors (blue, green, red, and yellow). The transitive pictures showed line drawings of transitive events. Half of these verification pictures matched with the corresponding sentences, whereas the other half differed from the corresponding sentences in one component of the event (e.g., the color of the possessum in genitive pictures).

We created 240 description pictures for the picture description tasks. Among these pictures, 40 were genitive pictures and 40 were transitive filler pictures. The remaining 160 pictures contained no objects, but two figurines, with one fully colored and the other in black and white. These pictures were designed to induce predicative expressions and were employed in the picture description tasks between two trials (e.g., De heks is groen [The witch is green]). 
We constructed 40 critical (genitive) trials and 40 filler (transitive) trials for each experiment list. We had two structures of the critical to-be-recalled sentences (s-genitive vs. of-genitive) and two recall cue conditions (cue vs. no cue). Eight counterbalanced pseudo-random lists were constructed so that in each critical sentence recall trial speakers had to recall an s-genitive sentence in four lists and an of-genitive sentence in the other four lists; the recall tasks appeared with a recall cue in four lists and with no cue in the other four. The item order was also counterbalanced between lists. All experimental factors were counterbalanced within items and participants.

\section{Procedure}

The experiment was conducted in a sound-attenuated room with a laptop. The stimulus presentation was controlled using E-Prime 2.0 and recorded by a Sony ICD-PX440 mp3 recorder. The experimenter first introduced the experiment. The participant was told that she would have to memorize sentences and should recall the exact sentence when she was signalled to do so. The participant then read the instruction and was familiarized with the tasks with four practice trials.

The genitive to-be-recalled sentences always preceded transitive filler tasks, and vice versa. The trials were separated by zero to two filler picture description tasks. The sequence of events of each critical trial was as follows (Figure 1): (1) a beep sound occurred simultaneously with the appearance of the to-be-recalled sentence. The participant silently memorized the sentence and pressed the spacebar when she finished; (2) a verification picture appeared on the screen. The participant pressed " 1 " on the numeric pad if she believed the sentence matched the picture and pressed " 2 " if not. The 
program automatically progressed to the next trial after either key was pressed; (3) a picture appeared on the screen, the participant described the picture using one simple sentence and pressed the spacebar; (4) the participant moved on to read the sentence illustrated on the screen and pressed the spacebar to continue; (5) the participant moved on to a filler picture verification task; (6) the second beep sound occurred, the participant recalled the previously memorized sentence, using the lexical cue on the screen when it was applicable. She pressed the spacebar after the response was finished.

\section{Scoring}

Responses for participants during the critical sentence recall task were first coded in terms of the recall of the message. The response was coded as a correct recall of the message if the utterance in the recall task encompassed a possessive relation, irrespective of which specific structure was used. We then coded the sentence structure of the responses as s-genitives, of-genitives, or 'others'. A response was coded as an s-genitive if the possessor preceded the possessum and the appropriate possessive morpheme (zijn for male possessor/haar for female possessor) was added between the possessor and the object. A response was coded as an of-genitive if the sentence began with the possessum, followed by the preposition van, and ended with the possessor. The rest of the responses, including omitted responses, were counted as 'other' responses.

\section{Results}

The data from one participant was excluded due to technical issues. The final data set contained 1600 target responses, among which were 442 s-genitive responses (27.6\%), 746 of-genitive responses (46.6\%), and 412 'other' responses $(25.7 \%)$. Among the 
'other' responses, $81.0 \%$ were failure to recall, partial recall (e.g., De eend... [The duck...]), or recall of a wrong sentence, $19.0 \%$ were the production of grammatical alternatives (e.g., predicative expressions such as De eend is rood [The duck is red]).

\section{Message recall}

The descriptive data and inferential analysis of verbatim recall accuracy in Experiment 1 and Experiment 2 as well as all the data and analysis scripts are available on the Open Science Framework (https://osf.io/yqwsj/). We first report the participants' accuracy in message recall, that is, the chance that the speakers successfully retrieved the possessive relation in the critical recall tasks. The overall accuracy of message recall was $74.3 \%$ (74.1\% in S-genitive condition and $75.1 \%$ in Of-genitive condition). The appearance of a lexical cue increased the accuracy by $19.0 \%$ (No cue $=65.1 \%$, Cue $=84.1 \%$ ). This difference was $20.0 \%$ when the to-be-recalled structure was an s-genitive and $18.0 \%$ when the to-be-recalled structure was an of-genitive. The participants' accuracy of message recall was fit by a Generalized Linear Mixed Model (GLM model), using the lme4 package in $\mathrm{R}$ (version 3.4.0). The model predicted the logit-transformed likelihood of a successful recall of the possessive relation of the to-be-recalled sentence. To-berecalled sentence structure and cue condition were included in the model as fixed factors. All predictors were entered into the model in a mean-centered form (i.e., sum contrast coded) (Schad, Vasishth, Hohenstein, \& Kliegl, 2020). For the analysis (and all the analyses thereafter), we employed the maximal random effects structure justified by the design (Barr, Levy, Scheepers, \& Tily, 2013). Specifically, we included the by-subject and by-item random intercepts as well as random slopes for all main effects and interactions in the fixed model. If the model failed to converge, we simplified the random 
model by first dropping the random correlations in one go, and then dropping one term at a time. The term dropping started from the most complex terms (usually an interaction term). When there were multiple terms with the same complexity, we compared the variances of the random effects in the last model and dropped the term that accounted for the least amount of variance first. We repeated this step until the model converged and no warning of singular fit was reported.

The model converged after dropping the by-subject and by-item random correlation. The summary of the fixed effects of the model is listed in Appendix A. The alpha was set at .05 . We found no significant main effects of to-be-recalled sentence structure $\left(\chi^{2}=\right.$ $0.192, \mathrm{df}=1, \mathrm{p}=.662)$. There was a significant main effect of the cue condition $(\chi 2=$ 100.72, df $=1, \mathrm{p}<.001)$, indicating the accuracy of message recall was higher in the Lexical cue condition. The two-way interaction between to-be-recalled sentence structure and the cue condition was not significant $\left(\chi^{2}=0.149\right.$, df $\left.=1, \mathrm{p}=.699\right)$.

\section{Proportion of S-genitive Responses}

We next report the results of the proportion of the sentence structures used in the critical sentence recall tasks. In this analysis, we excluded all the 'other' responses; we thus only examined the distribution of sentence structures in the valid responses. We first report the overall proportion of s-genitive responses as a function of cue condition (Figure 2a). The proportion of s-genitive responses was $33.8 \%$ in the Lexical cue condition and $44.0 \%$ in the No cue condition, resulting in a $10.2 \%$ guidance effect promoting the syntactic alternative of s-genitive (i.e., of-genitive). The proportion of s-genitive sentences as a function of to-be-recalled sentence structure and cue condition is reported in Table 1 . The 
proportion of s-genitive responses was $66.7 \%$ after an s-genitive to-be-recalled sentence and $8.8 \%$ after an of-genitive to-be-recalled sentence, resulting in a $57.9 \%$ structural priming effect. The priming effect was $51.5 \%$ in the Lexical cue condition and $67.9 \%$ in the No cue condition, resulting in a $-16.4 \%$ 'anti-lexical boost effect' on the persistence of the s-genitive structure (see Figure $2 b$ ).

\section{[Table 1 near here]}

\section{[Figure 2 near here]}

A GLM model was fit to predict the logit-transformed likelihood of an s-genitive response. To-be-recalled sentence structure and cue condition were included in the model as fixed factors. The final model included a random intercept and a random slope of tobe-recalled sentence structure for subjects as well as a random intercept for items. The random correlations were dropped. The summary of the fixed effects of the model is listed in Appendix A. As expected, we found a significant main effect of to-be-recalled sentence structure $(\chi 2=81.217, \mathrm{df}=1, \mathrm{p}<.001)$, indicating that speakers produced more s-genitive structures after an s-genitive to-be-recalled sentence. There was a significant main effect of the cue condition $(\chi 2=10.193, \mathrm{df}=1, \mathrm{p}=.001)$. The negative estimate of the fixed effect showed that the appearance of a possessum cue reduced the likelihood for speakers to produce an s-genitive sentence. We also found a significant two-way interaction between to-be-recalled sentence structure and cue condition $(\chi 2=4.684$, $\mathrm{df}=$ $1, \mathrm{p}=.030$ ), indicating the likelihood of the s-genitive production after an s-genitive vs. an of-genitive to-be-recalled sentence was smaller in the Lexical cue condition (Figure 2b). 


\section{Discussion}

The first finding of Experiment 1 is that a lexical cue facilitated message retrieval in the critical sentence recall tasks. Speakers were more likely to produce an utterance that specifies a possessive relation when they saw a lexical cue that repeated the possessum of the to-be-recalled sentence. This finding is consistent with previous studies that showed that the recall of the message of the sentence was improved by a lexical cue that was repeated from encoding to retrieval (e.g., Masson, 1979). More importantly, the lexical cue affected the likelihood for speakers to produce an s-genitive structure: fewer overall s-genitive responses were produced when a lexical cue, namely the possessum, was provided. Furthermore, the appearance of a lexical cue reduced the tendency for speakers to use an s-genitive structure after an s-genitive to-be-recalled sentence. Taken together, we found no evidence that the appearance of a lexical cue facilitates the retrieval of sentence structure in sentence recall when the lexical guidance promotes the alternative structure. This indicates that the strong guidance effect is detrimental to the effect of the lexical boost.

One might even claim that there was an "anti-boost" effect in Experiment 1. However, we suggest that this is an incidental by-product of the guidance effect. As the possessum cue biased the speakers to the of-genitive structure, it imposed an across-the-board effect that led to a higher likelihood of misremembering an s-genitive as an of-genitive. However, the chance of misremembering an of-genitive to-be-recalled sentence as an sgenitive sentence was already very low when there was no lexical cue, so the presentation of a lexical cue did not reduce this chance further. Thus the "anti-boost" effect seems to come about because there was little room for the cue to reduce s-genitive production after 
an of-genitive to-be-recalled sentence.

We have mentioned that another factor that reduces the lexical boost effect is the memory interference between encoding and retrieval. However, we argue that the memory interference itself did not eliminate the lexical boost. When no cue was provided to the participants, they showed a predominant tendency (76.9\%) to use an s-genitive structure after an s-genitive to-be-recalled sentence. Given that the s-genitive is the least frequent genitive structure in Dutch (van Bergen, 2011), this predominant production of sgenitives indicates that the participants successfully maintained and retrieved the memory traces of the s-genitive structure. The structure repetition should be driven by the memory of the sentence structure. This suggests that a memory-related precondition for the lexical boost effect to come about (i.e., the speakers maintain the structure of the encoded sentence) is still valid. So we argue that the lack of a lexical boost (or even the anti-boost effect) should not be exclusively attributed to the impaired memory of the to-be-recalled structure. The detrimental effect of a competitive constraint (lexical guidance) might also play a role.

In the second experiment, we adopted the same paradigm as the first experiment but with several important modifications. In Experiment 1, we only employed the phrasal head (i.e., the possessum) of the to-be-recalled sentence as a lexical cue. One consequence of such a design is that the more accessible lexical item could only guide speakers to produce of-genitives but not s-genitives. This might limit our understanding of the guidance effect induced by the memory cue. To have a more balanced assessment of the guidance effect, we cued both with the possessum and the possessor in Experiment 2. To do this, we enlarged the number of possessors of the genitive to-be-recalled sentences and 
varied the identities of these possessors so that no possessor was repeated within the experiment. We created a lexical cue corresponding to these possessors. This way, the lexical cue for the to-be-recalled sentence could be evenly distributed over the thematic roles. We predict that the cueing of either the possessum or the possessor should induce a guidance effect. Following the results of Experiment 1, we predict that the effect of the lexical boost might be weakened or even blocked by the guidance effect in Experiment 2 . In contrast, the 'anti-boost' effect is now not expected. This is because now that we have balanced the thematic role of the cue, the presence of a cue should no longer predispose the overall syntactic choice only to the of-genitive, as in Experiment 1 . Instead, the accessibility effect induced by a possessum cue and by a possessor cue should cancel each other out.

Many studies have shown that overlap in the head between the prime and target sentences magnifies structural priming (e.g., Cleland \& Pickering, 2003; Hartsuiker et al., 2008; Pickering \& Branigan, 1998; Scheepers et al., 2017). The prediction with regard to the boost of non-head overlap is less clear, but there is some evidence that non-head overlap facilitates structural priming (Scheepers et al., 2017; but see Carminati, van Gompel, \& Wakeford, 2019). Note that we did not set out to investigate the head-specificity of the lexical boost effect, so in most of the analyses we treat both possessum overlap and possessor overlap as lexical overlap, and the difference of thematic role on lexical overlap was only considered in some of the analyses.

Apart from to the change of experimental design, we adapted the procedure. We now excluded the picture verification task immediately after the sentence encoding task. This way, we ensured that the perception of the image did not interfere with the mental 
representation of the encoded to-be-recalled sentence.

\section{Experiment 2}

\section{Method}

Participants

Forty-eight Ghent University students, all native Dutch speakers, participated in exchange for course credit (38 females and 10 males, average age 19.31 years).

\section{Materials and Design}

The materials were similar to those of Experiment 1 but with several changes. First, we created 80 new genitive to-be-recalled sentences that varied in both the name of possessors and possessums (2a-2d). Twenty lexical cues were selected that corresponded to half of these possessors. Now, in each experimental list, the lexical cues were equally distributed between the possessor $(2 \mathrm{a}-2 \mathrm{~b}$, the underlined word was used as a lexical cue in the Lexical cue condition) and the possessum (2c-2d). Specifically, in ten critical trials of each list, the recall tasks were prompted by a lexical cue that corresponded to the possessum of the to-be-recalled sentence; in ten critical trials, the recall tasks were prompted by a lexical cue that corresponded to the possessors, and in the remaining twenty trials, no lexical cue was provided. Second, we excluded all the picture verification tasks. Instead, we inserted a recognition task after each filler picture description task and each filler sentence reading task, asking the participants to indicate whether they had seen the presented sentence or picture before (Figure 3). We duplicated 16 filler description pictures and 16 filler prime sentences and replaced the filler 
pictures/sentences in the original list with the duplicated stimuli. This way, $40 \%$ of the filler stimuli were repeated from previous trials. Finally, to increase the difficulty of the filler sentence recall task, we introduced a further variant of the transitive sentences, namely a PP-medial passive (e.g., De non wordt door de danser geduwd [Literally, The dancer is by the dancer pushed]). The three structures of the filler to-be-recalled task were counterbalanced within and between lists.

2a) De boer zijn tarwe is geel [Literally: The farmer his wheat is yellow]

2b) De tarwe van de boer is geel [Literally: The wheat of the farmer is yellow]

2c) De bakker zijn kaas is groen [Literally: The baker his cheese is green]

2d) De kaas van de bakker is groen [Literally: The cheese of the baker is green]

Similar to Experiment 1, we had a 2 (to-be-recalled sentence structure) x 2 (cue presentation) within-subject design. Eight pseudo-random lists were constructed that counterbalanced the structure of the to-be-recalled sentence (s-genitive vs. of-genitive), the appearance of a cue (Lexical cue vs. No cue), and the order of the items. The thematic role of the lexical cue (Possessum vs. Possessor) was counterbalanced within each list.

[Figure 3 near here]

\section{Procedure and Scoring}

The procedure was similar to that of Experiment 1 but with several differences (see Figure 3). First, after the filler picture description task, the participant moved on to a recognition task. The participant pressed " 1 " on the numerical pad if she believed that the 
picture that she just described had appeared in the previous trials. She pressed " 2 " otherwise. She went through a similar recognition task after the filler prime sentence reading task, in which she judged whether the filler prime sentence had appeared earlier on or not.

The scoring was the same as that in Experiment 1.

\section{Results}

The final data set contained 1920 target responses, among which were 607 s-genitive responses (31.6\%), 868 of-genitive responses (45.2\%), and 445 'other' responses (23.2\%). Among the 'other' responses, 59.5\% were failure to recall, partial recall, or recall of a wrong sentence, $40.5 \%$ were the production of grammatical alternatives.

\section{Message Recall}

We first report the participants' performance in message recall. The overall accuracy of message recall was $76.8 \%$ (74.4\% in S-genitive condition and $79.3 \%$ in Of-genitive condition). The appearance of a lexical cue increased the accuracy by $14.7 \%$ (Lexical cue $=84.1 \%$, No cue $=69.4 \%$ ). This difference was $19.2 \%$ when the to-be-recalled structure was s-genitive and $10.2 \%$ when the to-be-recalled structure was of-genitive. The participants' accuracy of message recall was fit by a GLM model. The model predicted the logit-transformed likelihood of a successful message recall. To-be-recalled sentence structure (sum contrast coded) and cue condition (sum contrast coded) were included in the model as fixed factors. The final model included a random intercept and a random slope of to-be-recalled sentence structure for subjects as well as a random intercept for 
items. The random correlations were dropped. The summary of the fixed effects of the model is listed in Appendix A. We found the main effect of to-be-recalled sentence structure $(\chi 2=5.115, \mathrm{df}=1, \mathrm{p}=.024)$, indicating speakers were less accurate in retrieving the message of an s-genitive sentence. More importantly, there was a significant main effect of cue condition $(\chi 2=70.582, \mathrm{df}=1, \mathrm{p}<.001)$, indicating the accuracy of message recall was improved in the Lexical cue condition. The interaction between to-be-recalled sentence structure and cue condition was significant $(\chi 2=4.319$, $\mathrm{df}=1, \mathrm{p}=.038$ ), indicating that the lexical cue exerted a stronger facilitation effect on the message recall of the s-genitive sentences.

\section{Proportion of S-genitive Responses}

We then consider the sentence structures used in the critical sentence recall tasks. Again, in this analysis, we excluded all the 'other' responses. We first report the proportion of sgenitive responses as a function of the cue thematic role (Figure $2 \mathrm{a}$ ). When a possessor cue was presented, the overall proportion of s-genitive production increased by $11.8 \%$, but when a possessum cue was shown it decreased by $2.6 \%$. We then report the proportion of s-genitive responses as a function of to-be-recalled sentence structure and cue condition (Table 1). The proportion of s-genitive responses was $78.9 \%$ after an sgenitive to-be-recalled sentence and $5.1 \%$ after an of-genitive to-be-recalled sentence. In the No cue condition, the s-genitive production after an s-genitive to-be-recalled sentence was $76.2 \%$ higher than that after an of-genitive to-be-recalled sentence. This difference was $72.4 \%$ in the Lexical cue condition (see Figure $2 b$ ).

We performed two inferential analyses to examine the effect of to-be-recalled sentence 
structure and lexical cue on the production of an s-genitive structure in a recall task. In the first analysis, we took the thematic role of the cue into account (Possessum vs. Possessor vs. No Cue) in the production of an s-genitive response. A GLM model was fitted that predicted the logit-transformed likelihood of an s-genitive response. To-berecalled sentence structure (sum contrast coded) and cue thematic role (sum contrast coded, No Cue as the reference level) were included in the model as fixed factors. The final model included a random intercept and a random slope of to-be-recalled sentence structure for subjects as well as for items. The random correlations were dropped. The summary of the fixed effects of the model is listed in Appendix A. We found a significant main effect of to-be-recalled sentence structure $(\chi 2=99.379, \mathrm{df}=1, \mathrm{p}<.001)$. There was a significant main effect of cue thematic role $\left(\chi^{2}=25.164\right.$, df $\left.=2, p<.001\right)$. The fixed effects showed that the likelihood of s-genitive production was significantly larger in the Possessor cue condition than in the No cue condition $(\beta=0.948, \mathrm{SE}=0.241, \mathrm{p}<.001)$, whereas this time no significant difference was found between the Possessum cue condition and the No cue condition $(\beta=-0.457, \mathrm{SE}=-1.489, \mathrm{p}=.137)$. There was no two-way interaction between to-be-recalled sentence structure and the cue thematic role $(\chi 2=1.857, \mathrm{df}=2, \mathrm{p}=.395)$

In the second analysis, we examined whether the to-be-recalled sentence structure (Sgenitive vs. Of-genitive) and the presentation of a cue (Cue vs. No cue) modulated the sgenitive production. A GLM model was fitted that predicted the logit-transformed likelihood of an s-genitive response. To-be-recalled sentence structure and cue condition (all sum contrast coded) were included in the model as fixed factors. The final model included a random intercept, a random slope of structure, and a random slope of cue 
condition for subject as well as for item (but with no random correlation). The summary

of the fixed effects of the model is listed in Appendix A. As expected, there was a significant main effect of to-be-recalled sentence structure $(\chi 2=97.100, \mathrm{df}=1, \mathrm{p}<.001)$. But neither the main effect of cue condition $\left(\chi^{2}=0.957, \mathrm{df}=1, \mathrm{p}=.328\right)$ nor the interaction between to-be-recalled sentence structure and cue condition was significant $(\chi 2=1.379, \mathrm{df}=1, \mathrm{p}=.240)$

\section{Combined Analysis of Experiment 1 and 2}

Next, in a combined analysis, we examined the lexical effects across the two experiments, which allows us to assess the guidance effects as well as the lexical boost effects with higher statistical power. Because only Experiment 2 employed the possessor as a cue, we excluded the data under the Possessor cue condition to avoid rank deficiency. A GLM model was fitted that predicted the logit-transformed likelihood of an s-genitive response. To-be-recalled structure, cue condition (Possessum cue vs. No cue), and experiment were included in the model as fixed factors (all sum contrast coded). The final model included a random intercept and a random slope of to-be-recalled structure for subjects as well as a random intercept, a random slope of to-be-recalled structure, and a random slope of cue condition for items. The random correlations were dropped. The summary of the fixed effects of the model is reported in Appendix A. To avoid redundancy, we only report the results of interest. There was a significant two-way interaction between to-be-recalled structure and experiment $(\chi 2=11.313, \mathrm{df}=1, \mathrm{p}$ $<.001$ ), indicating the participants in Experiment 2 had higher accuracy in recalling the sgenitive structure. This is possibly because that the possessor cue employed in Experiment 2 had an across-the-board effect such that the overall production of s- 
genitives was raised by the possessor cue. There was a main effect of cue condition $\left(\chi^{2}=\right.$ $7.345, \mathrm{df}=1, \mathrm{p}=.007)$. But the two-way interaction between cue condition and experiment was not significant $(\chi 2=0.253, \mathrm{df}=1, \mathrm{p}=.615)$, suggesting the effect of the possessum cue in Experiment 2 was not significantly different from that in Experiment 1. Neither the two-way interaction between to-be-recalled structure and cue condition $\left(\chi^{2}=\right.$ $0.484, \mathrm{df}=1, \mathrm{p}=.487)$ nor the three-way interaction between to-be-recalled structure and cue condition role and the experiment $(\chi 2=2.019, \mathrm{df}=1, \mathrm{p}=.155)$ was significant.

\section{Discussion}

In Experiment 2, we first replicated the lexical cueing effect on message retrieval in Experiment 1: Speakers were more likely to retrieve a genitive relation when a lexical cue is provided. More importantly, we partially replicated the guidance effect on the mapping from concept to the linear order of the sentence: Speakers were more likely to produce an s-genitive sentence when they saw a lexical cue that repeated the possessor of the to-be-recalled sentence. This is conceptually consistent with the finding in Experiment 1 . That is to say, the guidance effect does not entail that speakers put the more accessible word to the structural head of the NP. Rather, they arrange the linear order of the arguments in accordance with their accessibility, regardless of whether the arguments can be the head of the phrase structure. We failed to replicate the guidance effect of the possesum cue. Nevertheless, the combined analysis of Experiment 1 and 2 showed no significant difference in the effect of the possessum cue between the two experiments. The presence of a lexical cue did not boost the syntactic persistence in sentence recall, indicating once again that there might be a trade-off between lexical guidance and the lexical boost. 


\section{Experiment 3}

The findings of Experiments 1 and 2 jointly indicate that when a sentence is encoded in explicit memory, speakers do not necessarily exploit a lexical cue to facilitate the retrieval of the surface structure of the to-be-recalled sentence. Rather, lexical cues help the speakers to reinstate the message of the encoded sentence and guide the linearization process of sentence production.

In Experiment 1 and 2 we employed sentence recall tasks. In such tasks, the participants are instructed to consciously memorize sentences and accurately recall the same sentence later. This way, the flexibility of the speakers' syntactic choice might be constrained by the experiment design. This may limit the validity of our conclusions because it has been argued that syntactic processing in naturalistic speech often proceeds in a more automatic way (Levelt et al., 1999). To address this possibility, we sought to replicate the guidance effect found in Experiments 1 and 2 in a task that does not instruct the participants to utilize explicit memory (i.e., a structural priming task). We adopted the sentence readingsentence generation paradigm (Scheepers et al., 2017). In this paradigm, the speakers first read a sentence, and then produce a sentence using a triplet of word chunks presented on the screen. For instance, they might see Dutch equivalents of the chunks is yellow farmer - banjo and produce 'the farmer's banjo is yellow' in response. No explicit constraints were placed on the syntactic structure of the target sentence.

Different from the sentence recall task used in Experiments 1-2, the explicit presentation of a lexical cue prior to sentence production is not appropriate for a structural priming experiment. As memory retrieval is not explicitly required in this task, there is no reason 
to present an explicit memory cue. We therefore manipulated the accessibility of a lexical cue in a more implicit way. Analogous to Gleitman et al. (2007), we examined how selfgenerated attention shifts influenced speakers' syntactic choices. But while Gleitman et al. presented subtle visual cues, we exploited the spatial collocation of the lexical cue as a proxy of speakers' likelihood of the first fixation. Native speakers of Germanic languages usually read texts in a top-to-bottom, left-to-right direction, which likely leads to an initial fixation at the top and left region of the text (Just \& Carpenter, 1980; Rayner, Juhasz, \& Pollatsek, 2005). If the order in which speakers attend to the visual stimuli in our experiment is related to the way they formulate the sentence, one might expect a bias that prioritizes the processing of stimuli at the top and left area of the screen, leading to an effect on formulation that is analogous to the guidance effect. Prior work indeed showed that spatial collocation of the referents in a picture modulates speakers' syntactic choices (Hartsuiker, Kolk, \& Huiskamp, 1999; Pokhoday, Shtyrov, \& Myachykov, 2019): Speakers are more likely to start their utterance with the referent on the left side of the picture. In Experiments 3 and 4, the stimuli for the sentence generation task were three lexical chunks, displayed on the screen as a triangular lattice. We assumed that the chunk at the top of the screen would be more accessible than the two chunks at the bottom. If speakers exploit the lexical cue to guide the sentence linearization process in our structural priming task, they should be more likely to start the sentence with the lexical cue in the first row (i.e., the top chunk) of the arrays. Additionally, we manipulated whether there was lexical repetition (of the possessum or possessor) between the prime sentence and the target sentence. 


\section{Method}

\section{Participants}

Forty-eight further Ghent University students, all native Dutch speakers, participated in exchange for course credit (35 females and 13 males, average age 19.25 years). All participants reported to be non-color-blind and right-handed, and had normal or corrected-to-normal vision.

\section{Materials and Design}

A set of 432 prime sentences was created. The set consisted of 96 genitive sentences that alternated between s-genitive/of-genitive (2a-2d) and 144 transitive sentences that alternated between active/PP-medial passive/PP-final passive. Several of the genitive and transitive sentences were adopted from Experiment 2. The structure of the new genitive and transitive sentences was the same as that in Experiment 2. Another 192 intransitive prime sentences (e.g., De olifant stopt [The elephant stops]) were also constructed. These prime sentences were employed in the filler sentence reading tasks.

Corresponding to the genitive sentences ( $2 a-b$ and $2 c-d), 96$ word triplets were created (3a-b and 3c-d). The triplets consisted of a noun that denoted a person (e.g., boer [farmer]), a noun that denoted an object (e.g., banjo [banjo]), and a predicative chunk that was composed of the copula verb (is) and a color adjective (e.g., geel [yellow]). Half of the triplets repeated one argument noun of the prime sentence ( $3 a$ and $3 c$ ) and half of them did not repeat the argument noun ( $3 b$ and $3 d)$. Among the triplets that repeated an argument noun, half repeated the possessor of the prime sentence (3a) and half repeated 
the possessum of the prime sentence $(3 \mathrm{c})$. The color adjectives were always repeated between prime and target, so the term overlap condition thereafter specifically means the condition in which the nouns were repeated. Another 96 triplets were created that corresponded to transitive sentences employed in the filler sentence generation tasks. These triplets were composed of a noun that denoted the agent of the event, a noun that denoted the patient of the event, and a verb in the infinitive form (e.g., $\{$ kast, belemmeren, bus [cupboard, to obstruct, bus]\}). The agent/patient overlap between prime and target as well as the spatial order of the chunks were also manipulated.

3a) \{banjo, boer, is geel [banjo, farmer, is yellow]\}

3b) \{banjo, vreemde, is geel [banjo, stranger, is yellow]\}

3c) \{aannemer, kaas, is groen [builder, cheese, is green]\}

3d) \{aannemer, factuur, is groen [builder, invoice, is green]\}

Forty-eight critical trials were constructed for each experimental list. The stimuli in the sentence generation task consisted of three-word chunks each, displayed on the screen as a triangular lattice (see Figure 4). One component of the triplet was placed at the upper middle of the screen, and the other two were symmetrically placed at the lower left and lower right of the screen. Within each overlap condition, we also rotated the spatial order of the arrays so that each chunk was crossed with each spatial position of the triplet, thus leading to six display orders per triplet. The spatial order of the words in each of the triplets was randomly determined for each individual item within the experiment list and counterbalanced within each experiment list and between experiment lists. In two 
versions of the triplets, the possessor of the word triplets was placed at the top position of the lattice. In two versions of the triplets, the possessum was at the top position. In the remaining versions, the color adjective was placed at the top. Forty-eight filler trials were constructed in an analogous way with the transitive stimuli in the first sentence reading task and the sentence generation task. The critical trials and filler trials were interleaved and were separated by zero to two intransitive sentence reading trials.

We had a 2 (prime sentence structure) x 2 (overlap condition) x 3 (type of top chunk) within-subject design. Forty-eight pseudo-random lists were created that counterbalanced the prime sentence structure, the lexical overlap, the spatial position of the chunks, and item order within and between each list. The thematic role of the overlapping item was counterbalanced within each list.

[Figure 4 near here]

\section{Procedure}

Each trial began with a genitive sentence reading task, followed by an intransitive sentence reading task and ended with a genitive sentence generation task (see Figure 4). Participants were informed that the experiment involved two different tasks and that their spoken responses would be audio-recorded. The sentence reading task required the participants to read the sentence in silence. The sentence generation task required them to generate a concise and grammatical sentence using the words presented on the screen. Importantly, the participants were instructed to begin their sentences with any word on the screen. They were also informed that after the main task their performance would be evaluated in a brief posttest. They went through a practice session to ensure they were 
familiarized with the experimental procedure.

Following Scheepers et al. (2017), all the tasks in the experiment were computer-paced. In the critical sentence reading tasks, the sentence stimuli stayed on the screen for 5500 ms. The participant read the sentence in silence. Next, the filler sentence reading task lasted for $3500 \mathrm{~ms}$. After a $1000 \mathrm{~ms}$ presentation of a cross on the screen, the target word triplet was presented for $5500 \mathrm{~ms}$. The participants were instructed to generate a full sentence from each triplet, using all three elements of the triplet.

\section{Scoring}

The scoring of Experiment 3 is the same as Experiment 1.

\section{Results}

The final data set contained 2304 target responses, among which were 597 s-genitive responses (25.9\%), 1541 of-genitive responses (66.9\%), and 166 'other' responses (7.2\%). All analyses in Experiments 3 and 4 were conducted with the exclusion of the 'other' responses.

The descriptive data of the s-genitive production for each prime sentence structure $\mathrm{x}$ overlap thematic role $\mathrm{x}$ top chunk is illustrated in Table 2. First, we report the proportion of s-genitive production as a function of the thematic role of the lexical chunk that appeared at the top of the screen in the generation task (Possessor vs. Possessum vs. Predicative chunk). The proportion of s-genitives when the predicative chunk was the top chunk was $28.5 \%$, the proportion was $23.8 \%$ when the possessum was the top chunk, and $33.0 \%$ when the possessor was the top chunk (see Figure 5a). 


\section{[Table 2 near here]}

\section{[Figure 5 near here]}

Next, we report the effect of lexical overlap on the persistence of sentence structures. The descriptive data of the s-genitive production for each prime sentence structure $\mathrm{x}$ overlap condition is illustrated in Table 1. The proportion of s-genitive responses was 32.7\% after an s-genitive sentence and $24.0 \%$ after an of-genitive sentence, resulting in an $8.8 \%$ structural priming effect. When no argument was repeated between prime and target, the proportion of s-genitive responses after s-genitive primes was $7.4 \%$ higher than that after of-genitives. This difference was $9.9 \%$ when there was a lexical overlap (see Figure 5b).

To inferentially analyse the guidance effect, the lexical boost effect, and their possible interaction in Experiment 3, an omnibus test was conducted. A GLM model was fitted to predict the logit-transformed likelihood of an s-genitive response. The model examined whether the prime structure (S-genitive vs. Of-genitive), the type of top chunk (Possessum vs. Possessor vs. Predicate), and the overlap condition (Lexical overlap vs. No overlap) modulated the s-genitive production. The prime structure (sum contrast coded), the top chunk (sum contrast coded, Predicate as the reference level), and the overlap condition (sum contrast coded) were included in the model as fixed factors. The final model included a random intercept and a random slope of the prime sentence structure for subjects as well as a random intercept, a random slope of the prime sentence structure, and a random slope of the overlap condition for items. The random correlations were dropped.

The summary of the fixed effects of the model is listed in Appendix B. We found a 
significant main effect of prime structure $(\chi 2=17.696, \mathrm{df}=1, \mathrm{p}<.001)$, indicating that the proportion of s-genitive responses after an s-genitive prime was much larger than that after an of-genitive prime (i.e., a structural priming effect). There was a significant main effect of type of top chunk $\left(\chi^{2}=22.162, \mathrm{df}=2, \mathrm{p}<.001\right)$, suggesting that there were differences in the proportion of s-genitive responses that were induced by the three types of top chunks. The fixed effects of the type of top chunk (see Appendix B) showed that a more accessible possessum chunk induced fewer s-genitive responses than the predicative chunk $(\beta=-0.346, \mathrm{SE}=0.143, \mathrm{p}=.016)$ and a more accessible possessor chunk induced more s-genitive responses than the predicative chunk $(\beta=0.318, \mathrm{SE}=0.136, \mathrm{p}=.019)$. The two-way interaction between prime structure and overlap condition was not significant $\left(\chi^{2}=1.063, \mathrm{df}=1, \mathrm{p}=.303\right)$, showing no lexical boost effect on structural priming. There was a three-way interaction between prime structure, top chunk, and overlap condition $(\chi 2=9.790, \mathrm{df}=2, \mathrm{p}=.007)$. The fixed effects of the three-way interaction (see Appendix B) suggested that there was a significant lexical boost effect on structural priming when the possessum was placed at the top position $(\beta=1.879, \mathrm{SE}=$ $0.594, \mathrm{p}=.002)$.

\section{Discussion}

In Experiment 3, speakers' grammatical encoding of the sentence was affected by the accessibility of the target stimuli. Specifically, speakers tended to start the sentence with a chunk that was perceptually more salient. This indicates once again that they built up the sentence in a linearly incremental way, starting from the perceptually most accessible word. 
There was a significant structural priming effect on the production of s-genitives, replicating the priming effect of the same structure in Dutch-English bilingual speakers (Bernolet et al., 2013). Different from Bernolet et al. (2013), we did not find a significant effect of the lexical overlap on structural priming. One explanation for this lack of a lexical boost is once again related to memory interference exerted by a filler task between the prime and target tasks. However, as some authors have argued, the interference from a secondary sentence production task might not be detrimental to the memory maintenance of the prime structure (Branigan, Pickering, Stewart, et al., 2000). We suggest that although the filler task might disturb the memory maintenance of sentence structure, the interference should not be strong enough to entirely eliminate the lexical boost effect; rather the lexical boost dissipates as a consequence of the competition with the guidance effect.

Additionally, there was an interaction between the guidance and the lexical boost: Lexical overlap induced a boost effect, but only when the top chunk was the possessum. However, it is difficult to offer a sound theoretical interpretation of this result, and no such interaction was found in the rest of the experiments; we therefore refrain from speculation about this interaction.

\section{Experiment 4}

Here we examined whether speakers would be likely to exploit lexical overlap as a retrieval cue of sentence structure when they are instructed to attend to the syntactic structure. We therefore employed a sentence structure memory paradigm (Bernolet, Collina, \& Hartsuiker, 2016; Hartsuiker \& Kolk, 1998; Zhang et al., 2020). The 
procedure of this task very much resembled Experiment 3 except that speakers were instructed to maintain the memory of the prime sentence structure in order to reuse it in the target task. Different from the structural priming paradigm, the participants in a sentence structure memory experiment need to consider the accuracy in syntactic repetition, such that their sentence production in the target task was more constrained by the attention to the structure of the utterances. As mentioned in the introduction, we predict that when speakers pay more attention to a prime/to-be-recalled sentence, they will be guided more by the lexical-specific subcategorization structure and less by the lexical accessibility.

\section{Method}

\section{Participants}

Forty-eight further Ghent University students, all native Dutch speakers, participated in exchange for course credit (30 females and 18 males, average age 19.15 years). All participants reported to be non-color-blind and right-handed and had normal or correctedto-normal vision.

\section{Materials and Design}

The materials and design were the same as Experiment 3.

\section{Procedure}

The procedure was the same as Experiment 3 with an additional instruction for the memorization of the sentence structure. The participants were informed that in addition to the reading task and sentence generation task, a beep sound would accompany the 
sentence in some sentence reading tasks. When this happened, participants needed to memorize the structure of the sentence so as to reuse it in the upcoming generation tasks. After one intervening reading task, the second beep occurred, signalling the participants to reuse the memorized structure in the generation task. Before the test, the participants went through practice trials for them to react properly to the beep sounds.

\section{Scoring}

The scoring was the same as Experiment 3.

\section{Results}

The final data set contained 2304 target responses, among which were 964 s-genitive responses $(41.8 \%), 1258$ of-genitive responses (54.6\%), and 82 'other' responses $(3.6 \%)$.

We first report the effect of accessibility on the production of sentence structures (Table 2). The proportion of s-genitive production when the predicative chunk was the top chunk was $45.1 \%$, the proportion was $41.5 \%$ when the possessum was the top chunk, and $43.3 \%$ when the possessor was the top chunk (see Figure 5a). Next, we report the effect of lexical overlap on the persistence of s-genitive structures (Table 1). The proportion of sgenitive responses was $76.3 \%$ after an s-genitive sentence and $10.1 \%$ after an of-genitive sentence, resulting in a $66.2 \%$ memory effect. When no argument was repeated between the to-be-recalled sentence and the target, the proportion of s-genitive responses after an s-genitive to-be-recalled sentence was $62.9 \%$ higher than that after an of-genitive (see Figure 5b). This difference was $69.6 \%$ in the Lexical cue condition, indicating a $6.7 \%$ lexical boost effect on structure memory. 
A GLM model was fitted to predict the logit-transformed likelihood of an s-genitive response. The model examined whether to-be-recalled structure (S-genitive vs. Ofgenitive), type of top chunk (Possessum vs. Possessor vs. Predicate), and overlap condition (Lexical overlap vs. No overlap) modulated the s-genitive production. To-berecalled structure (sum contrast coded), top chunk (sum contrast coded, Predicate as the reference level), and overlap condition (sum contrast coded) were included in the model as fixed factors. The final model included a random intercept and a random slope of prime sentence structure for subjects as well as a random intercept, a random slope of prime sentence structure, and a random slope of overlap condition for items. The random correlations were dropped.

The summary of the fixed effects of the model is listed in Appendix B. We found a significant main effect of to-be-recalled structure $\left(\chi^{2}=72.192, \mathrm{df}=1, \mathrm{p}<.001\right)$, indicating that the proportion of s-genitive responses after an s-genitive prime was much larger than that after an of-genitive prime (i.e., a structure memory effect). The main effect type of top chunk was not significant $(\chi 2=4.238$, df $=2, p=.120)$. Nevertheless, when looking at the fixed effect of the type of top chunk (see Appendix B), the s-genitive responses were reduced when the possessum was placed at the top $(\beta=-0.346, \mathrm{SE}=$ $0.143, \mathrm{p}=.016)$. The two-way interaction between to-be-recalled structure and overlap condition was marginally significant $\left(\chi^{2}=3.376, \mathrm{df}=1, \mathrm{p}=.066\right)$. The three-way interaction between to-be-recalled structure, type of the top chunk, and overlap condition was not significant $(\chi 2=1.470, \mathrm{df}=2, \mathrm{p}=.480)$. Although the descriptive data seemed to suggest that the lexical boost effect was the strongest when the predicative chunk was placed at the top of the screen, the contrasts of lexical boost effect between the 
Predicative chunk and the rest of the chunks were not significant $\left(\mathrm{p}_{z} \mathrm{~s}>.1\right)$.

\section{Combined Analysis of Experiment 3 and 4}

Again, we conducted a cross-experiment analysis that combined the data of Experiment 3 and 4. We fitted a GLM model to predict the logit-transformed likelihood of an s-genitive response. The prime/to-be-recalled structure, type of top chunk (Predicate as the reference level), overlap condition, and experiment were included as fixed factors (all sum contrast coded). The final model included a random intercept, a random slope of structure and a random slope of type of top chunk for subjects as well as a random intercept, a random slope of structure, a random slope of overlap condition, and a random slope of type of top chunk for items. The fixed effects of the model are reported in Appendix B. Given the fixed effect model is rather complex, we only report the results of interest. There was a main effect of structure $(\chi 2=118.3, \mathrm{df}=1, \mathrm{p}<.001)$ and a significant interaction between structure and experiment $(\chi 2=79.858, \mathrm{df}=1, \mathrm{p}<.001)$. This suggests the tendency for the participants to repeat the syntactic structure was much stronger in Experiment 4. This is not surprising because in Experiment 4, the participants were instructed to repeat the experienced syntactic structure. The main effect of type of top chunk was significant $(\chi 2=7.597, \mathrm{df}=2, \mathrm{p}=.022)$. The fixed effects suggest this effect was mainly driven by the possessum cue. The two-way interaction between type of top chunk and the experiment was not significant $(\chi 2=3.556, \mathrm{df}=2, \mathrm{p}=.169)$. The interaction between structure and overlap condition was marginally significant $(\chi 2=$ $3.840, \mathrm{df}=1, \mathrm{p}=.050)$. The three-way interaction between structure, overlap condition, and the experiment was not significant $\left(\chi^{2}=0.330, \mathrm{df}=1, \mathrm{p}=.566\right)$. Taken together, the results indicate some evidence of both the guidance effect and the lexical boost effect. 
The difference between the two experiments, despite a numerical trend, was not significant.

\section{Discussion}

Experiment 4 replicated the guidance effect, at least partially. Speakers were less likely to produce an s-genitive sentence when the top chunk was the possessum. The combined analysis of Experiment 3 and 4 indicated that the possessum cue effect in Experiment 4 was not different from that in Experiment 3. But having the possessor as top chunk did not lead to an increase in the proportion of s-genitives. In contrast with the previous three experiments, there was a marginally significant lexical overlap effect in Experiment 4, suggesting that speakers might be able to use the repeated lexical item as a cue to facilitate the retrieving of sentence structure. Different from Experiment 3, there was no interaction between the lexical guidance and the lexical boost.

\section{General Discussion}

The current study examined the effects of lexical guidance and lexical boost on the syntactic encoding of Dutch genitives. In Experiments 1 and 2, speakers recalled Dutch genitive sentences with half of the trials accompanied by a lexical cue (the possessum in Experiment 1, the possessum or possessor in Experiment 2). In both experiments, the lexical cue facilitated the retrieval of genitive relations in sentence recall. In terms of syntactic decisions in sentence recall, presenting the possessum as a lexical cue significantly reduced the proportion of s-genitive productions in Experiment 1 . The lexical cue also showed an "anti-boost" effect: The structural priming effect was smaller if a possessum cue was given to the participants. In Experiment 2, presenting the 
possessor as a cue increased the likelihood that speakers produced an s-genitive, while there was also a trend that the possessum cue reduced s-genitive production. The presentation of a cue did not significantly affect the accuracy of s-genitive structure retrieval. There was no lexical boost effect or 'anti-boost' effect on sentence recall. In Experiment 3, speakers read Dutch genitive sentences and then generated genitive expressions from word triplets. The prime sentence structure and thematic overlap were manipulated. The accessibility manipulation was implemented by displaying either the name of the possessor or possessum at the top position of the screen, which we expected to attract initial visual attention. We found that the likelihood for speakers to produce an s-genitive structure increased when the possessor was placed at the visually salient location and decreased when the possessum was placed there. This replicated the guidance effects found in Experiments 1 and 2. Again, the lexical boost effect was not significant. Experiment 4 had the same design as Experiment 3, but we now instructed the participants to reuse the to-be-recalled sentence structure in the ensuing sentence production task. Presentation of a possessum cue reduced the proportion of s-genitives, but a possessor cue showed no effect. This time there was a marginal lexical boost effect on the persistence of s-genitive structure. In sum, four experiments using three paradigms found consistent evidence for the guidance effects on word order. Only in Experiment 4, using a sentence recall task, did we find evidence for a marginal lexical boost effect.

\section{Lexical Guidance Effects in Sentence Production}

All four experiments showed evidence for a guidance effect on the production of Dutch genitive structures. Speakers tended to choose a constituent structure in such a way that it placed the cued lexical item at the beginning of the sentence, irrespective of the structure 
of the to-be-recalled sentence or the prime sentence. The guidance effect occurred when a prime sentence structure is available for speakers to spontaneously retrieve (Experiment 3), when they were asked to encode and retrieve the sentence as a whole (Experiment 1 and 2), and even when they were specifically instructed to retrieve the sentence structure from the context (Experiment 4). The effect happened both when the lexical accessibility was high because the lexical item was presented as a cue word (Experiments 1-2) and when the lexical item appeared jointly with other words but in a visually more salient location (Experiments 3-4). There thus seems to be a near-ubiquitous effect of lexical guidance on sentence production.

To the best of our knowledge, our study is the first to find a guidance effect on the syntactic encoding of genitive structures. The finding is in keeping with previous studies that showed the guidance effects on the syntactic choices of transitives (Pokhoday et al., 2019; Prentice, 1967; Tannenbaum \& Williams, 1968; Turner \& Rommetveit, 1967, 1968), datives (Ferreira \& Yoshita, 2003; Myachykov et al., 2012), and joined NPs (Gleitman et al., 2007). The syntactic choice tested in the current study concerns phrasal structures (Dutch genitives) in which the nouns have the same grammatical function in the sentence (subject). Given that the word order alternation between s-genitive and ofgenitive does not involve the shift of grammatical roles, the choice between different genitives should tap into the level of positional encoding. Following Gleitman et al. (2007), the presentation of a lexical cue or the placement of the critical lexical item to the visually more salient region increased the accessibility of the cued item. This way, speakers could initiate a 'grab-and-go' process and start to plan the sentence from the perceptually most accessible words. The outcome of the conceptual encoding of the 
initial noun directly enters the morphosyntactic processing (i.e., the computation of a lemma representation, Levelt, Roelofs, \& Meyer, 1999) and eventually to physical articulation. The early placement of the more accessible words then arguably leads to an early articulation of the word.

Note that we do not argue that the sentence structure encoding is exclusively word-driven. Rather, the findings of the current study support the view that grammatical encoding is underpinned by an interaction between syntactic and lexical representations (Christianson \& Ferreira, 2005; Ferreira \& Dell, 2000). In all experiments, speakers’ syntactic choice was affected by the structure of the to-be-recalled or prime sentence preceding the target production (i.e., structural priming effects). Such effects were robust across different tasks and largely accounted for the variations in choices of genitive structures in most of the experiments. This is strong evidence that the structural assembly of Dutch genitives can be guided by a lexical-general structural relation that speakers just experienced. The co-existence of both the guidance effects and the structural priming effects in all four experiments indicates that the final outcome of grammatical encoding is not solely decided by the availability of the lexical items. Speakers do take into account both combinatorial information and non-combinatorial information when making syntactic decisions.

Following an interactive view of lexical-syntactic integration (Ferreira \& Dell, 2000), we argue that lexical accessibility does not simply guide the linearization of the sentence. Rather, it works along with structure-driven grammatical encoding in such a way that the selection among options in sentence structure building can be amenable to the accessibility of the words. In this view, lexical accessibility modulates the order in a 
phrase structure via syntactic choices that are also modulated by structural priming.

\section{Lexical Boost Effects in Sentence Recall and Structural Priming}

One main purpose of the current study was to investigate how a lexical cue functions in the persistence of syntactic structures. We first established that the presentation of a lexical cue did facilitate the retrieval of the conceptual representation of the encoded sentence. Speakers showed higher accuracy in the recall of the genitive relations (irrespective of whether it was an s-genitive or of-genitive) when the recall was aided by a lexical cue. Put differently, the presence of a lexical cue effectively reduced the chance that speakers produced an "other" response (mostly failure to recall or partial recall). This is in line with the previous findings that a cue that is identical or semantically related to the item from the to-be-recalled sentence facilitates the reinstatement of the conceptual relations in sentence recall (Masson, 1979; Till, 1977). Such a facilitation effect suggests that speakers do employ lexical-specific combinatorial information (conceptual relations in this case) in selecting the conceptual representations that they would retrieve in a recall task. Thus, we established that the lexical cue influences sentence formulation at least at the level of conceptual encoding.

We then examined whether a lexical cue or lexical overlap would facilitate the retrieval of the to-be-recalled (or primed) sentence structure. The current study is, to our knowledge, the first one to investigate the effect of lexical overlap (in the form of lexical cue) on the persistence of sentence structure in sentence recall tasks. Only in the sentence structure recall experiment (Experiment 4) did we find a marginal across-the-board lexical boost effect. The lexical boost was not significant in Experiment 2 and 3. In 
Experiment 1, in which only the head noun was used as a lexical cue, the cue even reduced the likelihood that speakers correctly retrieved s-genitive structures. Thus, the four studies reported here showed mixed evidence for lexical boost effects at best.

Before further discussing the lexical boost effects in the four experiments, it is necessary to first establish the likely mechanisms that account for the syntactic persistence in the sentence recall paradigm and the sentence structure memory paradigm. The structural priming experiment (i.e., Experiment 3) is the only one that allowed the participants to flexibly arrange the sentence. In the rest of the experiments, the participants had to consider whether the target utterance matched with the sentence they experienced. They had to be mindful of the accuracy of their responses. It is thus important to ask whether a sentence recall task entails grammatical encoding processes that are different from a structural priming task. More specifically, is there different weighing of the grammatical encoding components between the two tasks? To explore this question, we compared the magnitudes of the syntactic persistence effects between experiments. Although there were differences in certain methodological details, the designs and the type of target production of the structural priming experiment and the sentence recall experiments were similar. This allows us to compare the magnitudes of the sentence structure repetition between experiments. In Experiment 1 and 2, the likelihood of structure repetition was on average $65 \%$, which is much higher than that in Experiment $3(8.8 \%)$ and closer to that in the sentence structure memory tasks $(66.2 \%)$. This indicates that sentence production in a sentence recall task entails a stronger engagement of the explicit memory in comparison with the structural priming task. Such an assumption contrasts with a prevalent account of sentence recall which argued that syntactic persistence in sentence recall is strongly 
driven by structural priming (Potter \& Lombardi, 1998). We argue that the structural priming should not be the only source that underpins the syntactic persistence in a sentence recall task. Speakers rely strongly on the explicit memory of the encoded sentence to formulate the target sentence in a recall task.

Note that our assumption about the grammatical encoding process in sentence recall does not take away our prediction of a lexical boost effect in sentence recall. As we have argued, the lexical boost effect in structural priming can be attributed to a cueing effect in which the repeated item facilitates speakers to retrieve a syntactic structure from explicit memory (Chang, Dell, \& Bock, 2006; Hartsuiker et al., 2008; Zhang et al., 2020). Following this view, as long as the syntactic encoding in a production task involves conscious retrieval of the syntactic structure, a lexical boost effect is expected to occur. In this case, we would still expect that the lexical boost effect that strongly modulates structural priming should also influence the syntactic choice in sentence recall. If anything, we predicted that the lexical boost effect should be even stronger in the recall experiments, given that there is a more intense involvement of the sentence structure memory in the recall experiments. However, in the recall experiments as well as in the structural priming experiment, we found no evidence of lexical boost effects.

The absence of the lexical boost effects in Experiment 1-3 is inconsistent with previous studies that assessed the production of the structures under test. Bernolet et al. (2013) showed cross-linguistic effects of lexical overlap on genitive structural priming. In a within L2 experiment, Bernolet and colleagues showed that the structural priming effect was much larger when there was an overlap of the possessum (structural priming effect $=$ $80 \%$ ) than when there was no lexical overlap (structural priming effect $=48 \%$ ). We 
assumed that a similar lexical overlap effect should be found at least in the experiment that employed similar tasks as in Bernolet and colleagues (i.e., Experiment 3) and in the recall experiments. One possible reason for the inconsistent finding between the two studies is that the tasks used in the current study were different from those in Bernolet et al. (2013). The tasks employed in Experiment 3, although conceptually comparable to Bernolet et al. (2013), were different in the details of design and procedure (sentence reading-generation in Experiment 3 vs. dialogue task with scripted confederate in Bernolet et al.). Nevertheless, a study that employed the same task as in Experiment 3 showed considerable lexical boost effects of both head and non-head overlap on the priming of dative structures (Scheepers et al., 2017), suggesting the task is sensitive enough to induce a lexical boost effect on structural priming.

We have argued that there are a number of possible reasons for the lack of a consistent lexical boost in the sentence recall tasks and structural priming tasks. Among them, the lexical boost might be reduced by the memory interference of the filler tasks between sentence encoding and retrieval. Since the memory of sentence structure is only transient (Sachs, 1967), the effect of the lexical boost might also be amenable to the memory interference exerted by filler tasks (Hartsuiker et al., 2008). In our experiments, we inserted two fillers (Experiments 1 and 2) or one filler (Experiments 3 and 4) between sentence encoding and retrieval. The memory interference from the fillers might be detrimental to the memory maintenance of the sentence structure, such that the sentence structure became less retrievable upon a lexical cue. However, such an assumption does not seem to fit the results of Experiments 1 and 2. In both sentence recall experiments, we demonstrated that the lexical and structural traces of the to-be-recalled sentence did not 
diminish completely. Thus, the absence of lexical overlap effects on sentence structure retrieval in sentence recall should not be primarily attributed to the decay of the explicit memory. Here we argue that the occurrence, or lack thereof, of the lexical boost effect, might be at least partially driven by the competition between the lexical guidance and the lexical boost.

\section{Coordinating Lexical Information in Grammatical Encoding}

To summarize the findings above, in the current study we found that while the accessibility effects stayed robust in all four experiments, there was only a marginal lexical boost effect in some subsets of the data. The ubiquitous effect of lexical guidance and the null effects of lexical boost is analogous to the findings of Myachykov et al. (2012). Myachykov and colleagues manipulated factors including prime structure, lexical overlap, and the accessibility of the referents (via a visual cue) and examined the syntactic choices in producing transitive expressions (e.g., The cowboy is punching the boxer/The boxer is punched by the cowboy). They showed consistent effects of visual cueing on syntactic choices and an absence of the lexical boost effect at the same time. Given that, generally, the lexical overlap is by far the strongest modulator of structural priming and occurs for many different sentence structures (see Mahowald et al., 2016 for a meta-analysis of the magnitude of lexical boost effects on structural priming), it is important to discuss why we observed a null effect of lexical overlap on structural priming when the two arguments differ in lexical accessibility. We suggest that one of the reasons for the absence of the lexical boost effect in the face of the significant guidance effect might be the competition between the combinatorial information and the noncombinatorial information represented by the lexical items. 
Based on a rational view of sentence production, the final form of sentence production is jointly decided by various constraints that are sensitive to their reliability (Perek \& Goldberg, 2017; Thothathiri, 2021; Thothathiri \& Braiuca, 2021; Thothathiri \& Rattinger, 2016). As we have argued, the lexical guidance was the more reliable constraint in the current experiments, primarily because the more accessible item could lead to a one-toone mapping between the thematic role and the syntactic choice, whereas the lexical overlap could be mapped onto either one of the syntactic alternatives. If this is the case, the presence of the more reliable constraint (i.e., lexical guidance) might take precedence over the less reliable one (i.e., lexical boost) and predominantly modulates the syntactic choices in sentence production. In Experiment 1 and 2 of the study, the access of the lexical cue could simultaneously elicit a guidance effect and a lexical boost effect. In Experiment 3 and 4, the distribution of the accessibility between the arguments in the generation tasks was always unbalanced, thus it allowed the guidance effects to arise in all trials. This means that in all the conditions that the lexical boost effect could occur, the guidance effect could arise as well. Thus, the boost effects might be constantly overshadowed by the guidance effects while the guidance effect remained significant. This is exactly what we found in Experiment 1-3. Such an assumption could also explain the null effect of lexical overlap in Myachykov et al. (2012) in that the visual cue took precedence in grammatical encoding over other lexical-specific information and overshadowed the effect of the primed lexical-specific subcategorization frame.

It should be noted that there is one limitation of our study concerning the assessment of the rational view. In all of the experiments, the effect of lexical guidance always cooccurs with the condition that can induce a lexical overlap effect, which made it difficult 
to assess whether the lexical boost effect will arise when the lexical guidance is not available or even less available to the participants. A suggestion for future research would be to fully cross the occurrence of the lexical-specific effects or to vary their relative reliability. This way, we can have a better view of the conditions under which speakers could exploit the lexical-specific subcategorization frames. Putting the limitation aside, the findings of the study suggest that speakers rationally adapt the syntactic encoding to their language experience, based on the statistical distribution of the context.

Comparing the patterns of effects in Experiment 3 and Experiment 4, we suggest that attention to linguistic levels might play a role in the interplay between lexical boost and lexical guidance. There were certain differences in the distribution of the guidance effects and the lexical boost effects between Experiment 3 and 4. Specifically, whereas in Experiment 3 the guidance effects were robust for both the possessor and the possessum, in Experiment 4 the effect was only significant when the possessum was made more accessible. Meanwhile, a lexical boost (albeit a marginal one) was only found in Experiment 4. One interpretation of such differences in the patterns of effects is that in Experiment 3, the participants were not forced to reuse the sentence structure, thus the freedom in sentence formulation allowed the syntactic choices to be more amenable to lexical accessibility. When speakers were instructed to retrieve the sentence structure (Experiment 4), they tried to use the memory of the prime sentence to predict the structure of the target sentence. Thus, they were more motivated to attend to the memory of the lexical-specific subcategorization structure, leading to some effect of lexical overlap on syntactic choices. Furthermore, the participants in Experiment 4 would also be less likely to employ lexical accessibility to decide the syntactic structure, possibly 
because doing so would sometimes be detrimental to the reuse of sentence structure.

Thus, in Experiment 4, the attention to sentence structure partially overrode the reliability-related competition between constraints, which predisposed the participants to rely more on the lexical-specific subcategorization frame. We did not manipulate structural priming/structure memory in a within-subject design, so we could not make direct comparisons of the lexical effects in structural priming and sentence structure recall. The findings in Experiments 3 and 4 are only suggestive about the role of attention in grammatical encoding. It might be interesting for future studies to examine the mediating effect of attention on lexical processing in grammatical encoding via a withinsubject experiment that encompasses both paradigms of structural priming and structure recall.

\section{Conclusion}

Four experiments examined the role of lexical guidance and the lexical boost in the production of the Dutch genitive phrase structure. There was consistent evidence that speakers' syntactic choices in Dutch genitive production were guided by lexical cues, indicating the guidance effects on phrase encoding that are independent of functional processing. Significant effects of prime or to-be-recalled structures were also found in all four experiments. However, no evidence of a lexical boost was found, except when speakers were explicitly instructed to attend to the prime sentence structure. These findings support the view that speakers primarily exploit the non-combinatorial information of the lexical representations in grammatical encoding. The dependency of lexical-specific relational information might be reduced because of the competition with the guidance effect. 


\section{Notes}

1. Note that there is also a morphologically marked s-genitive in Dutch (e.g., Piet-s auto, where the $-s$ after the possessor Piet is a possessive morpheme). Nevertheless, the morphologically marked form in Dutch is only applicable to the possessive relations where the possessor is a proper noun (Scott, 2011; van Bergen, 2011; Weerman \& De Wit, 1999). The morphologically marked s-genitive was not employed in the present experiments.

\section{Funding details}

The research was supported by China Scholarship Council.

\section{Disclosure of Interest}

The authors report no conflict of interest

Informed consent was obtained for experimentation with human subjects.

\section{Data Availability Statement}

The data that support the findings of this study are openly available in Open Science Framework at http://doi.org/10.17605/OSF.IO/YQWSJ

\section{References}

Barr, D. J., Levy, R., Scheepers, C., \& Tily, H. J. (2013). Random effects structure for confirmatory hypothesis testing: Keep it maximal. Journal of Memory and Language, 68(3), 255-278. doi:https://doi.org/10.1016/j.jml.2012.11.001 
Bates, E., \& MacWhinney, B. (1982). Funetionalist approaches to grammar. In E. W. L. Gleitman (Ed.), Language acquisition: The state of the art (pp. 173-218). New York: Cambridge University Press.

Bates, E., \& MacWhinney, B. (1989). Functionalism and the competition model. In B. MacWhinney \& E. Bates (Eds.), The crosslinguistic study of sentence processing (Vol. 3, pp. 73-112). New York: Cambridge University Press.

Bernolet, S., Collina, S., \& Hartsuiker, R. J. (2016). The persistence of syntactic priming revisited. Journal of Memory and Language, 91, 99-116. doi:https://doi.org/10.1016/j.jml.2016.01.002

Bernolet, S., Hartsuiker, R. J., \& Pickering, M. J. (2012). Effects of phonological feedback on the selection of syntax: Evidence from between-language syntactic priming. Bilingualism-Language and Cognition, 15(3), 503-516. doi:10.1017/S1366728911000162

Bernolet, S., Hartsuiker, R. J., \& Pickering, M. J. (2013). From language-specific to shared syntactic representations: The influence of second language proficiency on syntactic sharing in bilinguals. Cognition, 127(3), 287-306. doi:https://doi.org/10.1016/j.cognition.2013.02.005

Bock, K. (1982). Toward a cognitive psychology of syntax: Information processing contributions to sentence formulation. Psychological review, 89(1), 1. doi:https://doi.org/10.1037/0033-295X.89.1.1

Bock, K. (1986). Meaning, sound, and syntax: Lexical priming in sentence production. Journal of Experimental Psychology: Learning, Memory, and Cognition, 12(4), 575. doi:https://doi.org/10.1037//0278-7393.12.4.575 
Bock, K. (1987). An effect of the accessibility of word forms on sentence structures. Journal of Memory and Language, 26(2), 119-137. doi:https://doi.org/10.1016/0749-596X(87)90120-3

Bock, K., \& Brewer, W. F. (1974). Reconstructive recall in sentences with alternative surface structures. Journal of experimental psychology, 103(5), 837. doi:https://doi.org/10.1037/h0037391

Bock, K., \& Irwin, D. E. (1980). Syntactic effects of information availability in sentence production. Journal of verbal learning and verbal behavior, 19(4), 467-484. doi:https://doi.org/10.1016/S0022-5371(80)90321-7

Bock, K., Irwin, D. E., \& Davidson, D. J. (2004). Putting first things first. In J. M. Henderson \& F. Ferreira (Eds.), The interface of language, vision, and action: Eye movements and the visual world (pp. 249-278). New York: Psychology Press.

Bock, K., \& Levelt, W. J. (1994). Language production: Grammatical encoding. In M. A. Gernsbacher (Ed.), Handbook of psycholinguistics (pp. 945-984). San Diego, CA: Academic Press.

Bock, K., \& Loebell, H. (1990). Framing sentences. Cognition, 35(1), 1-39. doi:https://doi.org/10.1016/0010-0277(90)90035-I

Bock, K., Loebell, H., \& Morey, R. (1992). From conceptual roles to structural relations: Bridging the syntactic cleft. Psychological review, 99(1), 150. doi:https://doi.org/10.1037/0033-295X.99.1.150 
Bock, K., \& Warren, R. K. (1985). Conceptual accessibility and syntactic structure in sentence formulation. Cognition, 21(1), 47-67. doi:https://doi.org/10.1016/0010$\underline{0277(85) 90023-X}$

Boland, J. E., \& Tanenhaus, M. K. (1991). The role of lexical representations in sentence processing. In G. B. Simpson (Ed.), Advances in psychology (Vol. 77, pp. 331366). North-Holland: Elsevier.

Branigan, H. P., \& McLean, J. F. (2016). What children learn from adults' utterances: An ephemeral lexical boost and persistent syntactic priming in adult-child dialogue. Journal of Memory and Language, 91, 141-157. doi:https://doi.org/10.1016/j.jml.2016.02.002

Branigan, H. P., Pickering, M. J., \& Cleland, A. A. (2000). Syntactic co-ordination in dialogue. Cognition, 75(2), B13-B25. doi:https://doi.org/10.1016/S0010$\underline{0277(99) 00081-5}$

Branigan, H. P., Pickering, M. J., Stewart, A. J., \& McLean, J. F. (2000). Syntactic priming in spoken production: Linguistic and temporal interference. Memory \& Cognition, 28(8), 1297-1302. doi:https://doi.org/10.3758/BF03211830

Carminati, M. N., van Gompel, R. P., \& Wakeford, L. J. (2019). An investigation into the lexical boost with nonhead nouns. Journal of Memory and Language, 108, 104031. doi:https://doi.org/10.1016/j.jml.2019.104031

Chang, F., Dell, G. S., \& Bock, K. (2006). Becoming syntactic. Psychological review, 113(2), 234. doi:https://doi.org/10.1037/0033-295X.113.2.234 
Christianson, K., \& Ferreira, F. (2005). Conceptual accessibility and sentence production in a free word order language (Odawa). Cognition, 98(2), 105-135. doi:https://doi.org/10.1016/j.cognition.2004.10.006

Cleland, A. A., \& Pickering, M. J. (2003). The use of lexical and syntactic information in language production: Evidence from the priming of noun-phrase structure. Journal of Memory and Language, 49(2), 214-230. doi:https://doi.org/10.1016/S0749-596X(03)00060-3

Dell, G. S., \& O'Seaghdha, P. G. (1994). Inhibition in interactive activation models of linguistic selection and sequencing. In D. Dagenbach \& T. H. Carr (Eds.), Inhibitory processes in attention, memory, and language. (pp. 409-453). San Diego, CA, US: Academic Press.

Deutsch, A., \& Dank, M. (2009). Conflicting cues and competition between notional and grammatical factors in producing number and gender agreement: Evidence from Hebrew. Journal of Memory and Language, 60(1), 112-143.

doi:https://doi.org/10.1016/j.jml.2008.07.001

Ferreira, F. (1994). Choice of passive voice is affected by verb type and animacy. Journal of Memory and Language, 33(6), 715-736. doi:https://doi.org/10.1006/jmla.1994.1034

Ferreira, V. S., \& Dell, G. S. (2000). Effect of ambiguity and lexical availability on syntactic and lexical production. Cognitive Psychology, 40(4), 296-340. doi:https://doi.org/10.1006/cogp.1999.0730 
Ferreira, V. S., \& Yoshita, H. (2003). Given-new ordering effects on the production of scrambled sentences in Japanese. Journal of psycholinguistic research, 32(6), 669-692. doi:https://doi.org/10.1023/A:1026146332132

Garrett, M. F. (1989). Processes in language production. Linguistics: The Cambridge survey, III. Language, Psychological and Biological Aspects, 69-96. doi:https://doi.org/10.1017/CBO9780511621062.004

Gleitman, L. R., January, D., Nappa, R., \& Trueswell, J. C. (2007). On the give and take between event apprehension and utterance formulation. Journal of Memory and Language, 57(4), 544-569. doi:https://doi.org/10.1016/j.jml.2007.01.007

Hartsuiker, R. J., Bernolet, S., Schoonbaert, S., Speybroeck, S., \& Vanderelst, D. (2008). Syntactic priming persists while the lexical boost decays: Evidence from written and spoken dialogue. Journal of Memory and Language, 58(2), 214-238. doi:https://doi.org/10.1016/j.jml.2007.07.003

Hartsuiker, R. J., \& Kolk, H. H. J. (1998). Syntactic facilitation in agrammatic sentence production. Brain and Language, 62(2), 221-254. doi:https://doi.org/10.1006/brln.1997.1905

Hartsuiker, R. J., Kolk, H. H. J., \& Huiskamp, P. (1999). Priming word order in sentence production. Quarterly Journal of Experimental Psychology Section a-Human Experimental Psychology, 52(1), 129-147.

doi:https://doi.org/10.1080/027249899391250

Haskell, T. R., \& MacDonald, M. C. (2003). Conflicting cues and competition in subjectverb agreement. Journal of Memory and Language, 48(4), 760-778. doi:https://doi.org/10.1016/S0749-596X(03)00010-X 
Ivanova, I., Pickering, M. J., Branigan, H. P., McLean, J. F., \& Costa, A. (2012). The comprehension of anomalous sentences: Evidence from structural priming. Cognition, 122(2), 193-209. doi:https://doi.org/10.1016/j.cognition.2011.10.013

Just, M. A., \& Carpenter, P. A. (1980). A theory of reading: From eye fixations to comprehension. Psychological review, 87(4), 329. doi:https://doi.org/10.1037/0033-295X.87.4.329

Kaplan, R. M., \& Bresnan, J. (1995). Lexical-functional grammar: A formal system for grammatical representation. In M. Dalrymple, R. M. Kaplan, J. T. Maxwell III, J. C. Maxwell, \& A. E. Zaenen (Eds.), Formal Issues in Lexical-Functional Grammar (pp. 29). Stanford, California: Center for the Study of Language (CSLI).

Kelly, M. H., Bock, J. K., \& Keil, F. C. (1986). Prototypicality in a linguistic context: Effects on sentence structure. Journal of Memory and Language, 25(1), 59-74. doi:https://doi.org/10.1016/0749-596X(86)90021-5

Konopka, A. E., \& Bock, K. (2009). Lexical or syntactic control of sentence formulation? Structural generalizations from idiom production. Cognitive Psychology, 58(1), 68-101. doi:https://doi.org/10.1016/j.cogpsych.2008.05.002

Levelt, W. J. (1989). Speaking: From intention to articulation. Cambridge, MA: MIT press.

Levelt, W. J., Roelofs, A., \& Meyer, A. S. (1999). A theory of lexical access in speech production. Behavioral and Brain Sciences, 22(1), 1-38. doi:https://doi.org/10.1017/S0140525X99001776 
Levin, B. (1993). English verb classes and alternations. Chicago: University of Chicago Press.

Lombardi, L., \& Potter, M. C. (1992). The regeneration of syntax in short term memory. Journal of Memory and Language, 31(6), 713-733. doi:https://doi.org/10.1016/0749-596X(92)90036-W

MacDonald, M. C. (2015). The emergence of language comprehension. The handbook of language emergence, 81-99. doi:https://doi.org/10.1002/9781118346136.ch3

Mahowald, K., James, A., Futrell, R., \& Gibson, E. (2016). A meta-analysis of syntactic priming in language production. Journal of Memory and Language, 91, 5-27. doi:https://doi.org/10.1016/j.jml.2016.03.009

Man, G., Meehan, S., Martin, N., Branigan, H., \& Lee, J. (2019). Effects of Verb Overlap on Structural Priming in Dialogue: Implications for Syntactic Learning in Aphasia. Journal of Speech, Language, and Hearing Research, 1-18. doi:https://doi.org/10.1044/2019 JSLHR-L-18-0418

Masson, M. E. (1979). Context and inferential cuing of sentence recall. Journal of verbal learning and verbal behavior, 18(2), 173-185. doi:https://doi.org/10.1016/S0022$\underline{5371(79) 90109-9}$

McDonald, J. L., Bock, K., \& Kelly, M. H. (1993). Word and world order: Semantic, phonological, and metrical determinants of serial position. Cognitive Psychology, 25(2), 188-230. doi:https://doi.org/10.1006/cogp.1993.1005

Melinger, A., Branigan, H. P., \& Pickering, M. J. (2014). Parallel processing in language production. Language, Cognition and Neuroscience, 29(6), 663-683. doi:https://doi.org/10.1080/23273798.2014.906635 
Melinger, A., \& Dobel, C. (2005). Lexically-driven syntactic priming. Cognition, 98(1), B11-B20. doi:https://doi.org/10.1016/j.cognition.2005.02.001

Myachykov, A., Garrod, S., \& Scheepers, C. (2009). Attention and syntax in sentence production: A critical review. Discours. Revue de linguistique, psycholinguistique et informatique, 4. doi:https://doi.org/10.4000/discours.7594

Myachykov, A., Garrod, S., \& Scheepers, C. (2010). Perceptual priming of structural choice during English and Finnish sentence production. Language \& cognition: State of the art, 54-72.

Myachykov, A., Garrod, S., \& Scheepers, C. (2012). Determinants of structural choice in visually situated sentence production. Acta Psychologica, 141(3), 304-315. doi:https://doi.org/10.1016/j.actpsy.2012.09.006

Myachykov, A., Scheepers, C., Garrod, S., Thompson, D., \& Fedorova, O. (2013). Syntactic flexibility and competition in sentence production: The case of English and Russian. Quarterly Journal of Experimental Psychology, 66(8), 1601-1619. doi:https://doi.org/10.1080/17470218.2012.754910

Myachykov, A., \& Tomlin, R. S. (2008). Perceptual priming and structural choice in Russian sentence production. Journal of Cognitive Science, 6(1), 31-48. doi:https://doi.org/10.17791/jcs.2008.9.1.31

Perek, F., \& Goldberg, A. E. (2017). Linguistic generalization on the basis of function and constraints on the basis of statistical preemption. Cognition, 168, 276-293. doi:https://doi.org/10.1016/j.cognition.2017.06.019 
Pickering, M. J., \& Branigan, H. P. (1998). The representation of verbs: Evidence from syntactic priming in language production. Journal of Memory and Language, 39(4), 633-651. doi:https://doi.org/10.1006/jmla.1998.2592

Pickering, M. J., Branigan, H. P., \& McLean, J. F. (2002). Constituent structure is formulated in one stage. Journal of Memory and Language, 46(3), 586-605. doi:https://doi.org/10.1006/jmla.2001.2824

Pokhoday, M. Y., Shtyrov, Y. Y., \& Myachykov, A. (2019). Effects of visual priming and event orientation on word order choice in Russian sentence production. Frontiers in Psychology, 10, 1661. doi:https://doi.org/10.3389/fpsyg.2019.01661

Potter, M. C., \& Lombardi, L. (1998). Syntactic priming in immediate recall of sentences. Journal of Memory and Language, 38(3), 265-282. doi:https://doi.org/10.1006/jmla.1997.2546

Prat-Sala, M., \& Branigan, H. P. (2000). Discourse constraints on syntactic processing in language production: A cross-linguistic study in English and Spanish. Journal of Memory and Language, 42(2), 168-182. doi:https://doi.org/10.1006/jmla.1999.2668

Prentice, J. L. (1967). Effects of cuing actor vs cuing object on word order in sentence production. Psychonomic science, 8(4), 163-164. doi:https://doi.org/10.3758/BF03331600

Rayner, K., Juhasz, B. J., \& Pollatsek, A. (2005). Eye movements during reading. The science of reading: A handbook, 79-97. doi:https://doi.org/10.1002/9780470757642.ch5 
Rowland, C. F., Chang, F., Ambridge, B., Pine, J. M., \& Lieven, E. V. (2012). The development of abstract syntax: Evidence from structural priming and the lexical boost. Cognition, 125(1), 49-63.

doi:https://doi.org/10.1016/j.cognition.2012.06.008

Sachs, J. S. (1967). Recognition memory for syntactic and semantic aspects of connected discourse. Perception \& Psychophysics, 2(9), 437-442. doi:https://doi.org/10.3758/BF03208784

Schad, D. J., Vasishth, S., Hohenstein, S., \& Kliegl, R. (2020). How to capitalize on a priori contrasts in linear (mixed) models: A tutorial. Journal of Memory and Language, 110, 104038. doi:https://doi.org/10.1016/j.jml.2019.104038

Scheepers, C. (2003). Syntactic priming of relative clause attachments: Persistence of structural configuration in sentence production. Cognition, 89(3), 179-205. doi:https://doi.org/10.1016/S0010-0277(03)00119-7

Scheepers, C., Raffray, C. N., \& Myachykov, A. (2017). The lexical boost effect is not diagnostic of lexically-specific syntactic representations. Journal of Memory and Language, 95, 102-115. doi:https://doi.org/10.1016/j.jml.2017.03.001

Scott, A. K. (2011). The position of the genitive in present-day Dutch. Word structure, 4(1), 104-135. doi:https://doi.org/10.3366/word.2011.0005

Stallings, L. M., MacDonald, M. C., \& O'Seaghdha, P. G. (1998). Phrasal ordering constraints in sentence production: Phrase length and verb disposition in heavyNP shift. Journal of Memory and Language, 39(3), 392-417. doi:https://doi.org/10.1006/jmla.1998.2586 
Tanaka, M. N., Branigan, H. P., McLean, J. F., \& Pickering, M. J. (2011). Conceptual influences on word order and voice in sentence production: Evidence from Japanese. Journal of Memory and Language, 65(3), 318-330. doi:https://doi.org/10.1016/j.jml.2011.04.009

Tannenbaum, P. H., \& Williams, F. (1968). Generation of active and passive sentences as a function of subject or object focus. Journal of verbal learning and verbal behavior, 7(1), 246-250. doi:https://doi.org/10.1016/S0022-5371(68)80197-5

Thothathiri, M. (2021). Rational and Flexible Adaptation of Sentence Production to Ongoing Language Experience. Frontiers in Psychology, 12, 894. doi:https://doi.org/10.3389/fpsyg.2021.647076

Thothathiri, M., \& Braiuca, M. C. (2021). Distributional learning in English: The effect of verb-specific biases and verb-general semantic mappings on sentence production. Journal of Experimental Psychology: Learning, Memory, and Cognition, 47(1), 113. doi:https://doi.org/10.1037/xlm0000814

Thothathiri, M., \& Rattinger, M. G. (2016). Acquiring and producing sentences: Whether learners use verb-specific or verb-general information depends on cue validity. Frontiers in Psychology, 7, 404. doi:https://doi.org/10.3389/fpsyg.2016.00404

Till, R. E. (1977). Sentence memory prompted with inferential recall cues. Journal of Experimental Psychology: Human Learning and Memory, 3(2), 129. doi:https://doi.org/10.1037/0278-7393.3.2.129

Turner, E. A., \& Rommetveit, R. (1967). Experimental manipulation of the production of active and passive voice in children. Language and Speech, 10(3), 169-180. doi:https://doi.org/10.1177/002383096701000303 
Turner, E. A., \& Rommetveit, R. (1968). Focus of attention in recall of active and passive sentences. Journal of verbal learning and verbal behavior, 7(2), 543-548. doi:https://doi.org/10.1016/S0022-5371(68)80047-7

van Bergen, G. (2011). Who's first and what's next. Animacy and word order variation in Dutch language production (Unpublished doctoral dissertation) Radboud University, Nijmegen.

Vigliocco, G., \& Hartsuiker, R. J. (2002). The interplay of meaning, sound, and syntax in sentence production. Psychological Bulletin, 128(3), 442-472. doi:10.1037//00332909.128.3.442

Weerman, F., \& De Wit, P. (1999). The decline of the genitive in Dutch. doi:https://doi.org/10.1515/ling.37.6.1155

Zhang, C., Bernolet, S., \& Hartsuiker, R. J. (2020). The role of explicit memory in syntactic persistence: Effects of lexical cueing and load on sentence memory and sentence production. Plos One, 15(11), e0240909. doi:https://doi.org/10.1371/journal.pone.0240909

Ziegler, J., Bencini, G., Goldberg, A., \& Snedeker, J. (2019). How abstract is syntax? Evidence from structural priming. Cognition, 193, 104045. doi:https://doi.org/10.1016/j.cognition.2019.104045 


\section{Tables}

\section{Table 1}

Proportions s-genitive responses out of all s-genitive and of-genitive responses for each prime condition $\mathrm{x}$ cue condition combination in each experiment

\begin{tabular}{|c|c|c|c|c|}
\hline \multirow[b]{2}{*}{ Experiment } & \multirow[b]{2}{*}{ Cue/overlap condition } & \multicolumn{2}{|c|}{ To-be-recalled structure } & \multirow{2}{*}{$\begin{array}{l}\text { Structure } \\
\text { repetition }\end{array}$} \\
\hline & & S-genitive & Of-genitive & \\
\hline \multirow{2}{*}{1} & No cue & .769 & .090 & .679 \\
\hline & Lexical cue & .596 & .081 & .515 \\
\hline \multirow{2}{*}{2} & No cue & .796 & .035 & .761 \\
\hline & Lexical cue & .793 & .069 & .724 \\
\hline \multirow{2}{*}{3} & No overlap & .333 & .258 & .075 \\
\hline & Lexical overlap & .320 & .221 & .099 \\
\hline \multirow{2}{*}{4} & No overlap & .737 & .108 & .629 \\
\hline & Lexical overlap & .790 & .094 & .696 \\
\hline
\end{tabular}

Note. "S-genitive" and "of-genitive" in the header row indicate the structures of the to-be-recalled sentence. The "Structure repetition" in the third header row indicates the priming effect (the proportion of s-genitive responses in s-genitive prime condition minus that in of-genitive prime condition). 


\section{Table 2}

Proportions and of s-genitive responses out of all s-genitive and of-genitive responses for each prime condition $\mathrm{x}$ cue thematic role $\mathrm{x}$ type of top chunk combination in Experiment 3 and 4.

\begin{tabular}{|c|c|c|c|c|c|c|c|c|c|c|}
\hline \multirow{3}{*}{ Experiment } & \multirow{3}{*}{$\begin{array}{c}\text { Thematic role } \\
\text { overlap }\end{array}$} & \multicolumn{9}{|c|}{ Top chunk } \\
\hline & & \multicolumn{3}{|c|}{ Predicate } & \multicolumn{3}{|c|}{ Possessor } & \multicolumn{3}{|c|}{ Possessum } \\
\hline & & S & Of & $\begin{array}{l}\text { Structure } \\
\text { repetition }\end{array}$ & $S$ & Of & $\begin{array}{l}\text { Structure } \\
\text { repetition }\end{array}$ & S & Of & $\begin{array}{l}\text { Structure } \\
\text { repetition }\end{array}$ \\
\hline \multirow[t]{2}{*}{3} & No overlap & .357 & .227 & .129 & .367 & .296 & .070 & .246 & .289 & -.043 \\
\hline & Lexical overlap & .298 & .237 & .061 & .388 & .307 & .081 & .306 & .131 & .176 \\
\hline \multirow[t]{2}{*}{4} & No overlap & .745 & .128 & .617 & .735 & .103 & .632 & .745 & .097 & .649 \\
\hline & Lexical overlap & .830 & .108 & .722 & .788 & .115 & .674 & .745 & .086 & .659 \\
\hline
\end{tabular}

Note. "S" and "OF" in the third header row indicate the prime structure (s-genitive and ofgenitive). The "Structure repetition" in the third header row indicate the priming effect in Experiment 3 and sentence memory effect in Experiment 4 (the proportion of s-genitive responses in s-genitive prime condition minus that in of-genitive prime condition). 
Figures

\section{Figure 1}

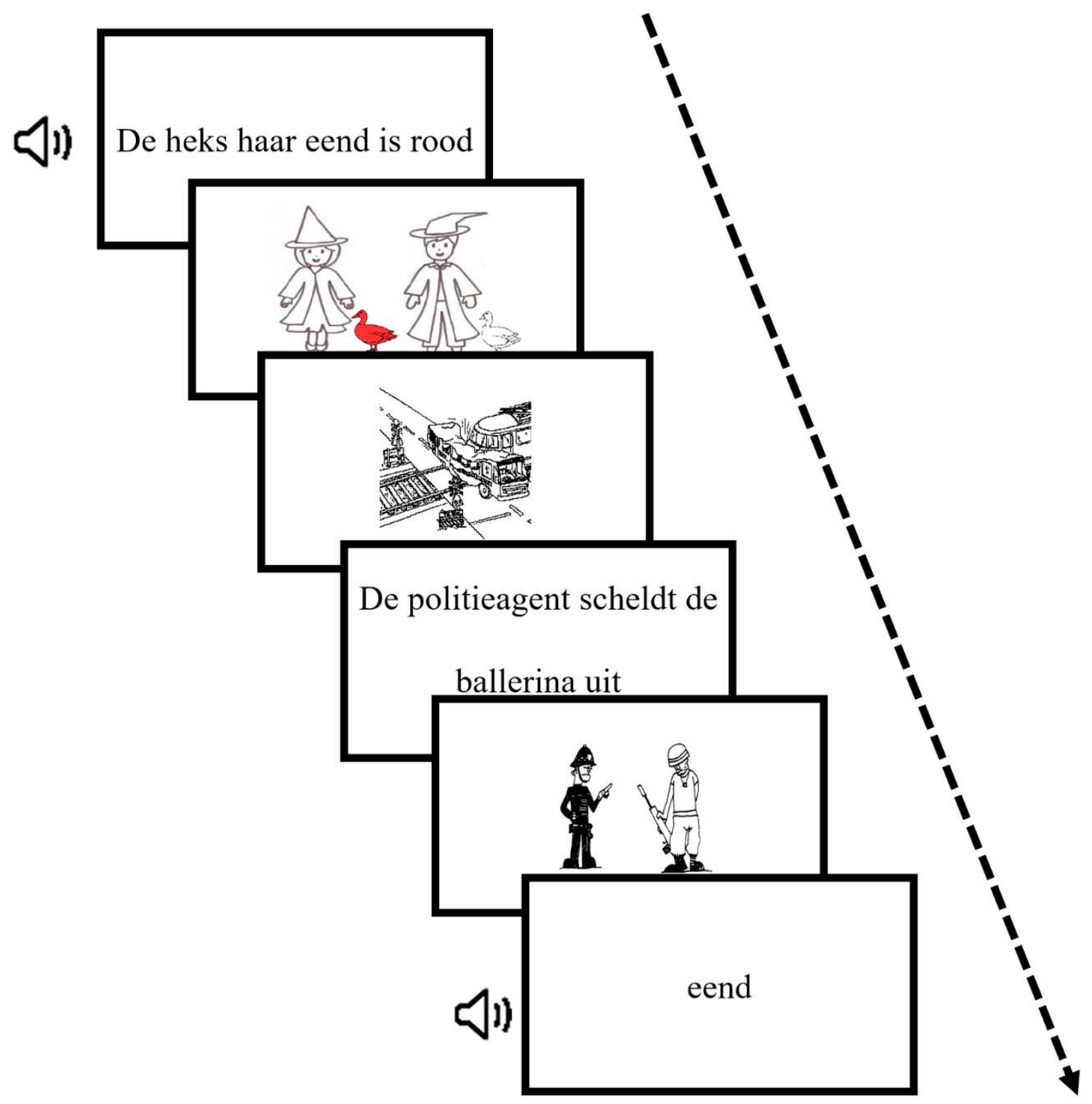




\section{Figure 2}

(a)

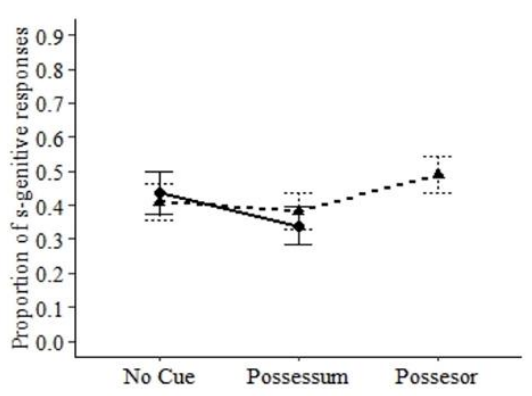

(b)

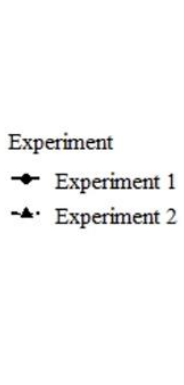

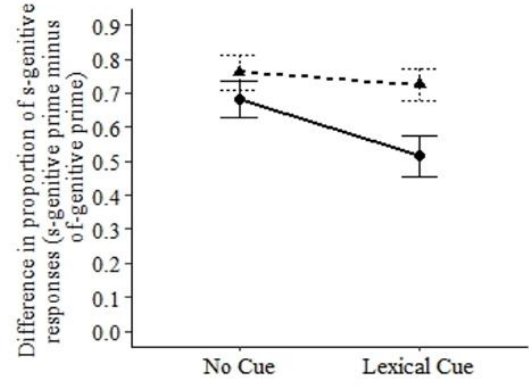

Experiment

- Experiment 1

-1. Experiment 2 
Figure 3

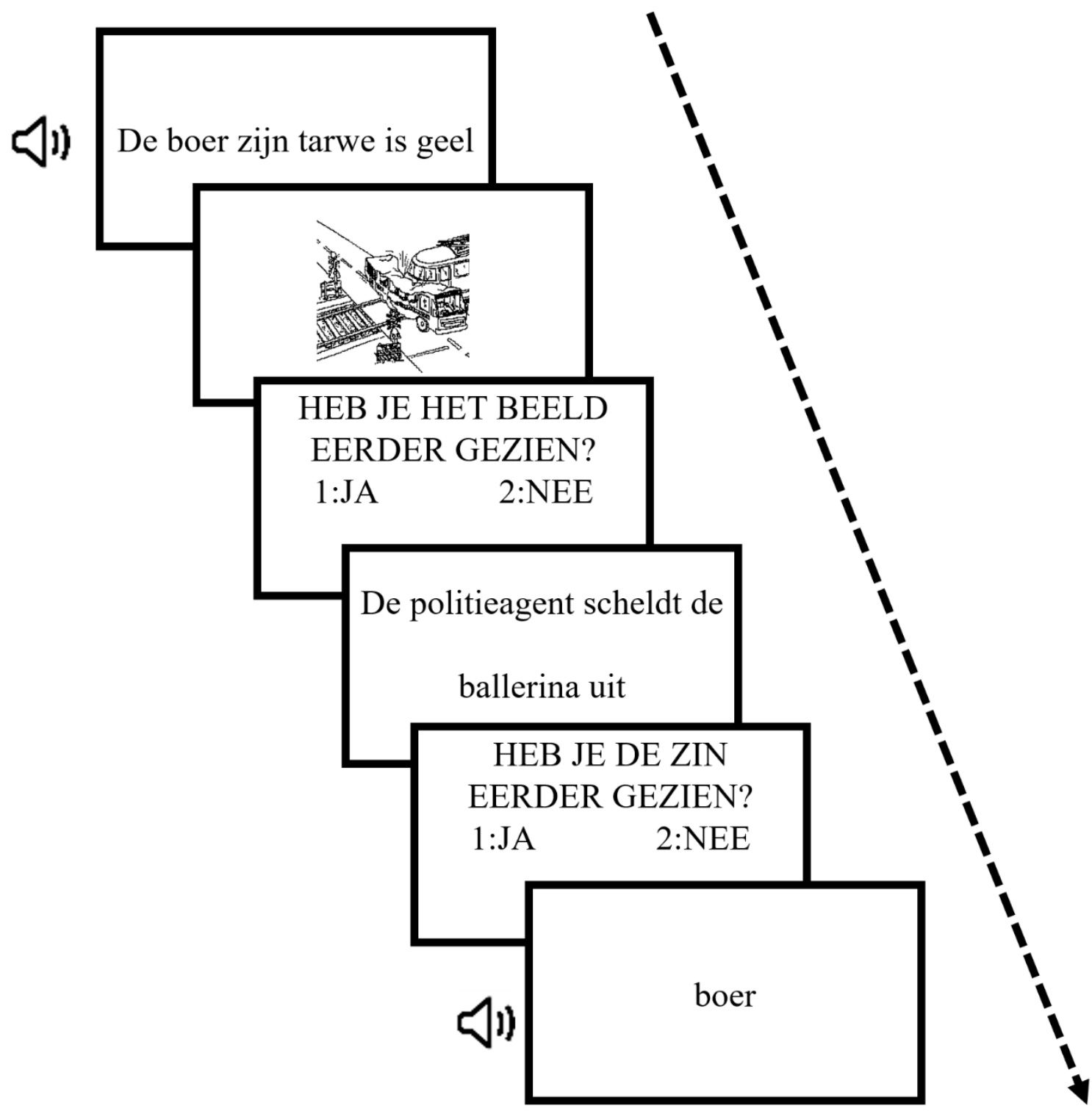




\section{Figure 4}

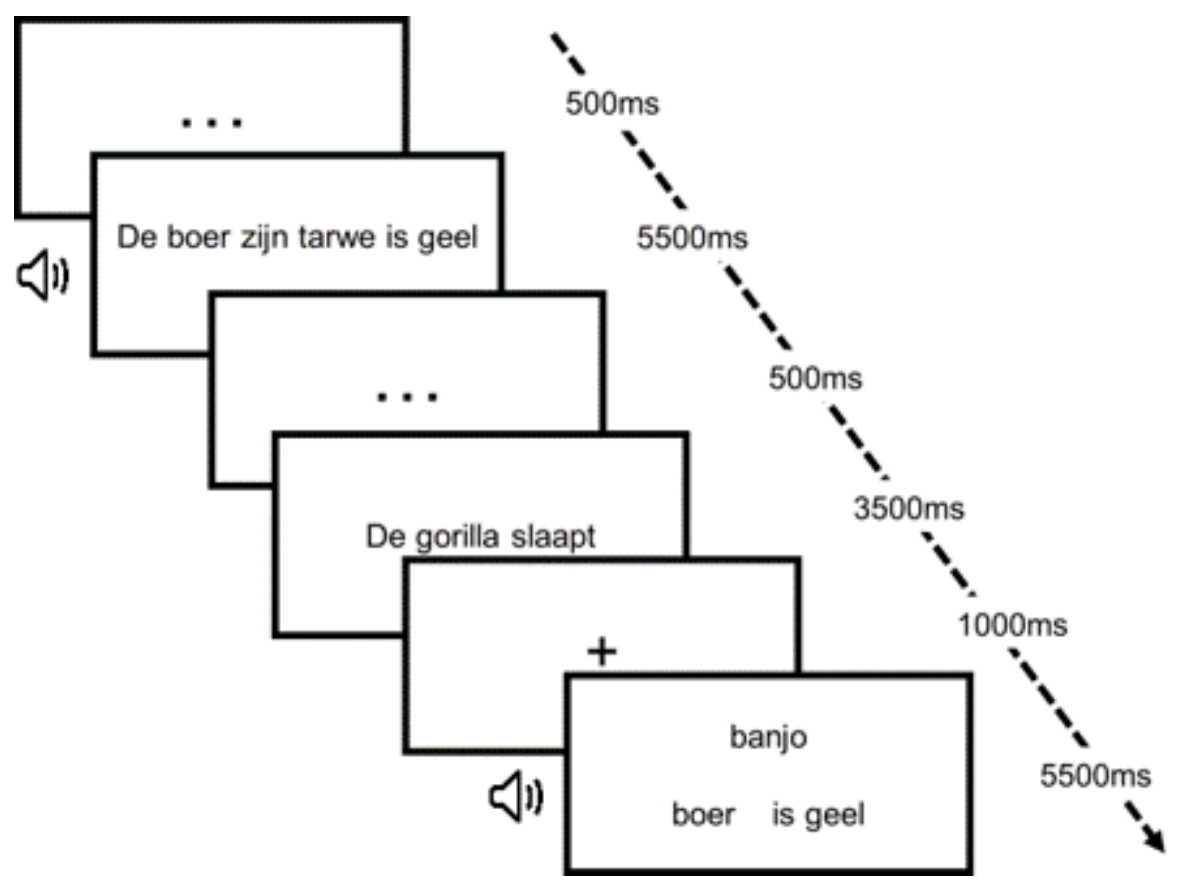




\section{Figure 5}

(a)

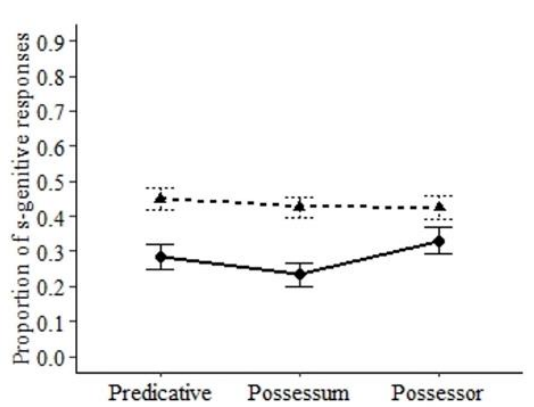

(b)

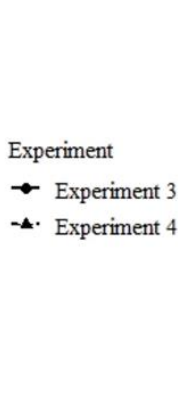

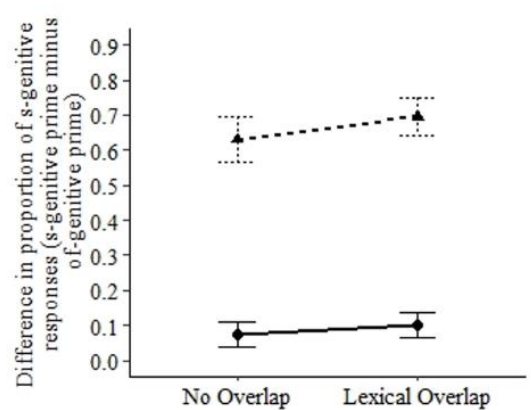

Experiment

- Experiment 3

-.. Experiment 4 


\section{Figure Captions}

Figure 1 The procedure of Experiment 1. Each trial for participants consisted of the following events: A critical sentence memorization task, a picture verification task, a picture description task, a sentence reading task, a picture verification task, and finally a sentence recall task (with a lexical cue). The tone symbol signals sentence memorization and recall.

Figure 2 (a) The proportion of s-genitive responses as a function of the thematic role of the cue in Experiment 1-2; and (b) The priming effect (s-genitive production in the sgenitive condition minus that in the of-genitive condition) as a function of cue condition in Experiment 1- 2

Figure 3 The procedure of Experiment 2. Each trial for participants consisted of the following events: A critical sentence memorization task, a picture description task, a picture recognition task, a sentence reading task, a sentence recognition task, and finally a sentence recall task (with a lexical cue). The tone symbol signals for sentence memorization and recall. Question in the picture recognition task (the third picture): Heb je het beeld eerder gezien? [Have you seen the picture before?]. Question in the sentence recognition task (the fifth picture): Heb je de zin eerder gezien? [Have you seen the sentence before?].

Figure 4 The procedure of Experiments 3 and 4. Each trial for participants consisted of the following events: After a $500 \mathrm{~ms}$ interval the prime sentence was presented for 5500 ms. After another $500 \mathrm{~ms}$ interval a filler sentence reading task was presented $(3500 \mathrm{~ms})$, followed by a $1000 \mathrm{~ms}$ interval and a finally a target triplet $(5500 \mathrm{~ms})$. The tone symbol signals sentence structure memorization and recall in Experiment 4.

Figure 5 (a) The proportion of s-genitive responses as a function of the type of the top chunk in Experiment 3-4; and (b) the priming effect (s-genitive production in the sgenitive condition minus that in the of-genitive condition) as a function of lexical overlap in Experiment 3-4. 